\title{
Experimental Study of Bump Effects on Boundary-Layer Transition in Compressible High Reynolds Number Flow
}

\author{
M Costantini ${ }^{1 *}$, S Risius ${ }^{1}$, S Koch ${ }^{1}$, C Fuchs ${ }^{1}$, U Gerhard ${ }^{1}$, S Hein ${ }^{1}$ and C Klein ${ }^{1}$ \\ ${ }^{1}$ DLR, Institute of Aerodynamics and Flow Technology, Göttingen, Germany \\ *Marco.Costantini@dlr.de
}

\begin{abstract}
The influence of surface bumps on boundary-layer transition was experimentally investigated in the present work. The experiments were conducted in a (quasi-) two-dimensional flow at low to high subsonic Mach numbers and chord Reynolds numbers up to 10 million in the low-turbulence Cryogenic LudwiegTube Göttingen. Various streamwise pressure gradients relevant for natural laminar flow surfaces were examined. Quasi-two-dimensional bumps, with a sinusoidal shape in the streamwise direction, fixed length and three different heights, were installed on a two-dimensional flat-plate model. The model was equipped with temperature-sensitive paint for non-intrusive transition detection and with pressure taps for the measurement of the surface pressure distributions. Boundary-layer transition was shown to occur at a more upstream location with increasing bump height-to-length ratio. This was mainly due to the local adverse pressure gradient on the downstream side of the bump, which was particularly pronounced in the case of the bump with the largest height-to-length ratio, thereby inducing boundary-layer separation (as verified via oil-film visualizations). In the case of the bump with the smallest height-to-length ratio, bumpinduced transition was found to be dependent on global pressure gradient, Mach number and Reynolds number; however, the influence of these parameters on transition induced by bumps with larger height-tolength ratios was significantly reduced. The sensitivity of boundary-layer transition to the effect of the bumps was shown to be more pronounced with stronger global flow acceleration and at smaller Mach numbers.
\end{abstract}

\section{Introduction}

Natural Laminar Flow (NLF) technology has been demonstrated to be a powerful tool for drag reduction on aircraft surfaces with zero to moderate sweep angles, where the amplification of streamwise instabilities is the predominant mechanism leading to transition - see Holmes and Obara (1983), Wagner et al. (1989) and Arnal (1992). The drag reduction capability of NLF technology has been verified for a wide range of aircraft classes, from sailplanes to business jets and transport aircraft, as reported in, e.g., Holmes and Obara (1983), Wagner et al. (1989) and Hansen (2010). One of the major concerns about the practicability of NLF technology for aircraft drag reduction is related to the achievability of surface smoothness compatible with NLF requirements. Surface imperfections such as steps, gaps, bumps, waviness, incorrectly installed flush rivets, etc., can affect aircraft surfaces, as discussed in Nayfeh et al. (1988) and Hansen (2010). Already in Holmes and Obara (1983) it had been shown that modern manufacturing techniques for metallic and composite materials can now provide surface smoothness suitable for laminar flow; however, manufacturing tolerances must be specified for the shape and dimension of surface imperfections so that laminar flow can still be achieved. As emphasized in Nayfeh et al. (1988) and Hansen (2010), a guide is needed for size and shape of surface imperfections in order to specify manufacturing tolerances as precisely as possible, thus avoiding overly stringent requirements.

The present work focuses on surface bumps. They can occur, as reported by Wagner et al. (1989) and Hansen (2010), at or between structural joints, ribs, and stringers and in the region of flush rivets. In fact, 
Revision 1. Submitted to the Special Issue of Experimental Thermal and Fluid Science (ETFS) dedicated to the $5^{\text {th }}$ International Conference on Experimental Fluid Mechanics (ICEFM)

significant waviness may be found on modern aircraft surfaces only in the form of (widely) spaced bumps. Criteria for allowable tolerances can thus be developed for this type of imperfection and then extended to multiple bumps.

The influence of bumps on boundary-layer stability and transition has been investigated for specific surface geometries and flow conditions in earlier numerical and experimental work. It was already observed in Hood (1939) that a nearly-sinusoidal bump, placed at approximately $10 \%$ of the chord length of a NACA 23012 airfoil model, induced premature transition and thus a significant drag increase. These experiments were conducted at Mach numbers up to 0.6 and chord Reynolds numbers up to $17 \cdot 10^{6}$. The earliest systematic experimental study on the effects of surface imperfections on boundary-layer transition was reported in Fage (1943). The experiments were carried out at low speed (Mach numbers of approximately 0.1 or less) on two-dimensional flat plates with a slightly accelerated flow. The "critical" imperfection size was defined as the minimal height $h$ of the imperfection "which affects the location of transition", i.e., which leads to a (measurable) upstream shift of the transition location, as compared to that on the smooth surface. The correlation of the results provided the following relation to establish the critical imperfection height:

$$
h / a=9 \cdot 10^{6}\left(\operatorname{Re}_{x \mathrm{~T}}\right)^{-1.5}\left(x_{\mathrm{T}} / a\right)^{0.5}
$$

where $a$ is the length of the imperfection, while $x_{\mathrm{T}}$ is the transition location and $\operatorname{Re}_{x \mathrm{~T}}$ the transition Reynolds number (i.e., the Reynolds number based on the transition location) obtained on the bump-free surface. The critical heights of surface imperfections determined with Eq. (I) were also seen to be in agreement with the results of low-speed wind-tunnel experiments on an EQH 1260 airfoil model, while the agreement with data from (unpublished) flight tests was fair. Note that the shape of the imperfection and the streamwise pressure gradient were considered as "relatively unimportant parameters".

Single and multiple (closely spaced) sinusoidal waves were examined in the experimental studies of Carmichael (1959) on laminar surfaces with wall suction. Two series of experiments were conducted at "low to moderate local Mach numbers": on a $30^{\circ}$ swept wing, investigated in three different wind tunnels, and on an unswept glove, installed on a wing of a Lockheed F-94A aircraft and tested in flight. Unfortunately, only sparse details on these experiments were provided in Carmichael (1959). In the unswept glove flight tests, the waves were placed in a non-suction region, but the boundary layer was strongly accelerated. A "critical" wave was defined in Carmichael (1959) "as the minimum size which prevents the attainment of laminar flow to the trailing edge [of the wing] under moderate suction". The criterion to determine the critical imperfection size proposed in this work was:

$$
h / a=244 \operatorname{Re}^{-0.75}(a / c)^{-0.5}
$$

where $h$ and $a$ are here the double amplitude and the wavelength of the imperfection, respectively, Re the chord Reynolds number and $c$ the chord length. A modification of Eq. (II) was also provided in Carmichael (1959) to account for the influence of wing sweep, according to the results obtained in the swept-wing experiments.

A series of numerical investigations between the late 1980's and the mid 1990's focused on the effect of bumps on the stability of flat-plate boundary layers at zero pressure gradient. The effect of the imperfections on transition was predicted according to the $\mathrm{e}^{\mathrm{N}_{\mathrm{T}}}$ method (see Arnal (1992)), where $\mathrm{N}_{\mathrm{T}}$ is the amplification factor ( $\mathrm{N}$-factor) of Tollmien-Schlichting waves at the transition location. (The value of $\mathrm{N}_{\mathrm{T}}$ was taken from earlier correlations with experimental data on smooth surfaces.) The contour of the imperfection was a quadratic and/or a cubic function of the streamwise coordinate. An incompressible flow was examined in Nayfeh et al. (1988) and in Cebeci and Egan (1989). In these studies and in the investigations cited below, the increase in the amplification factors of Tollmien-Schlichting waves due to the influence of the bump was found to be small for low values of the bump height-to-length ratio $h / a$, at 
Revision 1. Submitted to the Special Issue of Experimental Thermal and Fluid Science (ETFS) dedicated to the $5^{\text {th }}$ International Conference on Experimental Fluid Mechanics (ICEFM)

which the boundary layer remained attached over the bump surface. In contrast, the bump-induced disturbance amplification was markedly enhanced in the presence of a separated flow region on the downstream side of higher bumps. The effect of wall cooling on the bump-induced disturbance amplification was examined in Al-Maaitah (1990) for a Mach number of 0.8. In the considered cases, boundary-layer separation occurred on the downstream side of the bump. Because of the counteracting effects of a non-adiabatic surface in the attached and separated flow regions, an optimal ratio between surface and adiabatic-wall temperatures $T_{w} / T_{a w}$ was found to exist with regard to boundary-layer stability; the optimal value of $T_{w} / T_{a w}$ depended on $h / a$ and on the bump location. The effect of changes in various parameters ( $h / a$, Mach number, unit Reynolds number and bump location, among others) on boundarylayer stability was examined in Masad and Nayfeh (1992), Masad and Iyer (1993) and Masad and Malik (1995), where bumps with both attached and separated flows were considered. The results of these parametric investigations will be discussed later in this work, where they will be compared with the present experimental results. A more recent experimental investigation was reported in Perraud et al. (2004), where the effect of a nearly-sinusoidal bump on transition was examined for an accelerated flow on an NLF supercritical airfoil model, tested at a Mach number of 0.65 . Even bumps with relatively small $h / a$ were seen to induce a significant upstream shift of the transition location. Moreover, a model for the bump-induced variation of the $\mathrm{N}$-factors was proposed based on linear stability theory and developed for an incompressible flow at zero pressure gradient. The model was shown to provide a satisfactory prediction of the movement of the transition location observed in the aforementioned experiment. The effect of the bump shape (for fixed bump height) on boundary-layer stability was examined in Masad and Iyer (1993), Wörner et al. (2002), Perraud et al. (2004) and Franco et al. (2018). The general conclusion from these numerical studies was that the bump-induced increase in the $\mathrm{N}$-factors of Tollmien-Schlichting waves was larger for fuller bumps, i.e., for bumps with a shape tending to that of a rectangular bump. In particular, the size (in both streamwise and wall-normal directions) of the separated flow region on the downstream side of the super-Gaussian bumps, which were examined in Franco et al. (2018) for a flow at a Mach number of 0.5 and a zero pressure gradient, was shown to increase for fuller bumps. Moreover, the bump-induced change in the boundary-layer disturbance growth was found to be localized, so that - from a streamwise location sufficiently downstream of the bump location - the slope of the $\mathrm{N}$-factor envelope curves in the presence of the bumps was similar to that of the boundary layer over the smooth surface. At a sufficient distance from the bump location, the difference between the two $\mathrm{N}$-factor envelope curves can be reasonably assumed to be a uniform offset, which value was shown to depend on the shape of the imperfection.

The analysis of previous work shows that criteria for allowable bump size, covering a wide range of boundary-layer stability situations, are still not available. In particular, the effect of a variation in the streamwise pressure gradient on bump-induced transition has not been systematically examined up to now. This systematic investigation was carried out in the present work, where bump-induced transition was examined for various streamwise pressure gradients in a compressible flow at high Reynolds numbers. Sinusoidal bumps were installed on a two-dimensional flat plate and studied in the Cryogenic Ludwieg-Tube Göttingen (DNW-KRG). The experiments were conducted in a (quasi-) two-dimensional flow at freestream Mach numbers from 0.35 to 0.77 and chord Reynolds numbers up to $10 \cdot 10^{6}$. The test conditions are relevant for NLF surfaces with zero to moderate sweep angles. The effect of the bumps on boundary-layer transition was investigated by means of the Temperature-Sensitive Paint (TSP) technique, while their effect on the surface pressure distribution was measured using pressure taps. Oil-film visualizations (OFV) were conducted for selected test conditions to verify the occurrence of flow separation on the downstream side of the bumps. 
Revision 1. Submitted to the Special Issue of Experimental Thermal and Fluid Science (ETFS) dedicated to the $5^{\text {th }}$ International Conference on Experimental Fluid Mechanics (ICEFM)

\section{Experimental Setup}

\subsection{Cryogenic Ludwieg-Tube Göttingen (DNW-KRG)}

The experiments were conducted in the Cryogenic Ludwieg-Tube Göttingen, a low-turbulence facility (momentum turbulence level $\mathrm{Tu}_{\rho u} \sim 0.06 \%$ - see Koch (2004)) with the peculiarity of using nitrogen as test gas. A sketch of DNW-KRG is presented in Figure 1: dark and light blue areas show the facility components where the gas can be charged to the desired pressure and temperature values, and to where they are kept at atmospheric conditions, respectively. As discussed in Rosemann (1997), the values of Reynolds and Mach numbers characteristic of transport aircraft cruising at high subsonic or transonic speeds can be matched in DNW-KRG by increasing the pressure and decreasing the temperature of gaseous nitrogen. The test section dimensions are $0.4 \times 0.35 \times 2 \mathrm{~m}^{3}$ (width $\times$ height $\times$ length). The top and bottom walls of the test section are adaptive, and thus allow interference-free contours to be set (see Rosemann (1997)). Two-dimensional (i.e., spanwise-invariant) models with chord length $c \leq 0.2 \mathrm{~m}$ are clamped into turntables mounted at the side walls of the test section. Details on the wind-tunnel instrumentation and on its accuracy are given in Rosemann (1997), Koch (2004) and Costantini et al. (2016).

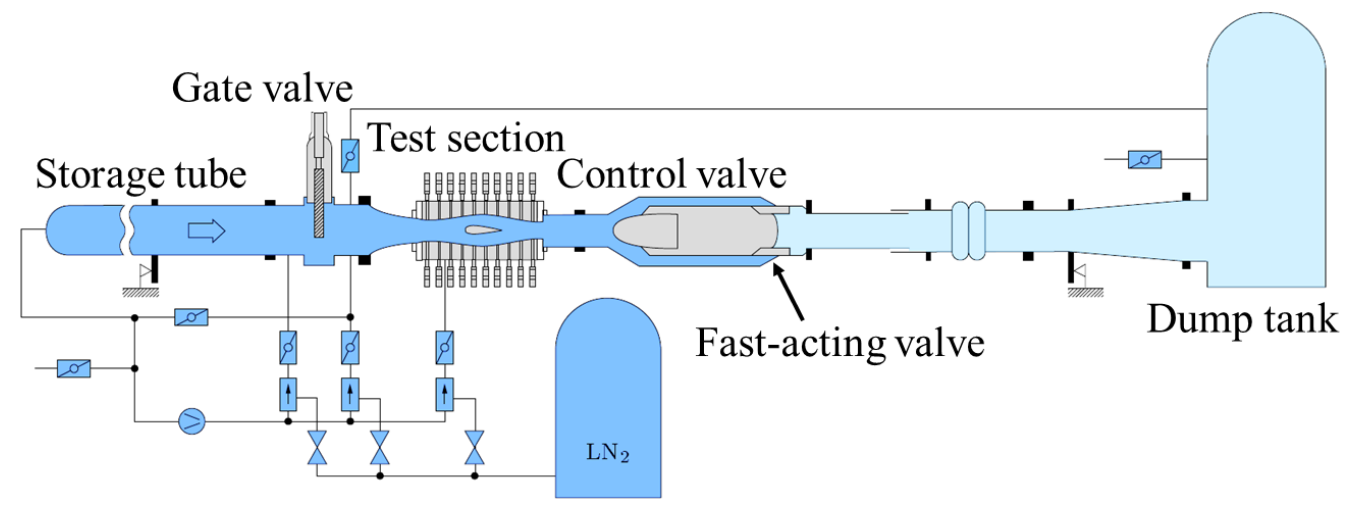

Figure 1: Principle sketch of the DNW-KRG wind tunnel (Koch (2004)).

The maximal testing time at DNW-KRG is $1 \mathrm{~s}$, attainable by the use of a very long storage tube (130 m). In the present work, the testing time was approximately $0.75 \mathrm{~s}$. During a run, the flow in the test section is stationary for some tenths of a second; however, previous work of Costantini (2016) has shown that the boundary-layer evolution over the model surface is stable only for a time window of approximately $0.2 \mathrm{~s}$ at the end of the run time. In the present work, all data were averaged over an evaluation window of $0.1 \mathrm{~s}$ at the end of the run time.

\subsection{Wind-Tunnel Model and Experimental Techniques}

The two-dimensional wind-tunnel model BuLASTra (Bump modification of the PaLASTra model) used in the experiments is shown in Figure 2. An available flat-plate configuration, presented in Costantini et al. (2016) and Costantini (2016), was modified to install exchangeable inserts on the model upper side. The leading-edge part was indeed the same for both investigations, while the model main part was modified for the present study. The surface bumps were manufactured on the inserts and had a sinusoidal shape in the streamwise direction $x$, given by the following equation:

$$
z(\xi)=\frac{h}{2}\left(\cos \left(\frac{2 \pi \xi}{a}\right)+1\right) \quad \text { for }-\frac{a}{2} \leq \xi \leq \frac{a}{2}
$$


Revision 1. Submitted to the Special Issue of Experimental Thermal and Fluid Science (ETFS) dedicated to the $5^{\text {th }}$ International Conference on Experimental Fluid Mechanics (ICEFM)

where $a$ is the streamwise extent of the bumps, $h$ the height of the bump crest and $\xi=x-x_{\mathrm{b}}$ the streamwise coordinate relative to the bump crest location $x_{\mathrm{b}}$. In the present work, the streamwise extent of the bumps was $a=20 \mathrm{~mm}$, i.e., $10 \%$ of the model chord $c=200 \mathrm{~mm}$. The start and the end of the bumps in the streamwise direction were located at $x / c=40$ and $50 \%$, respectively, with the bump crest being located at $x_{\mathrm{b}} / c=45 \%$. The bumps were quasi-two-dimensional: their spanwise extent was ten times larger than their streamwise extent, as sketched in Figure 2b. The present experimental setup enabled an independent variation of Mach number, chord Reynolds number, streamwise pressure gradient and bump height, and thus allowed to decouple the effects of changes in the aforementioned parameters on boundary-layer transition.
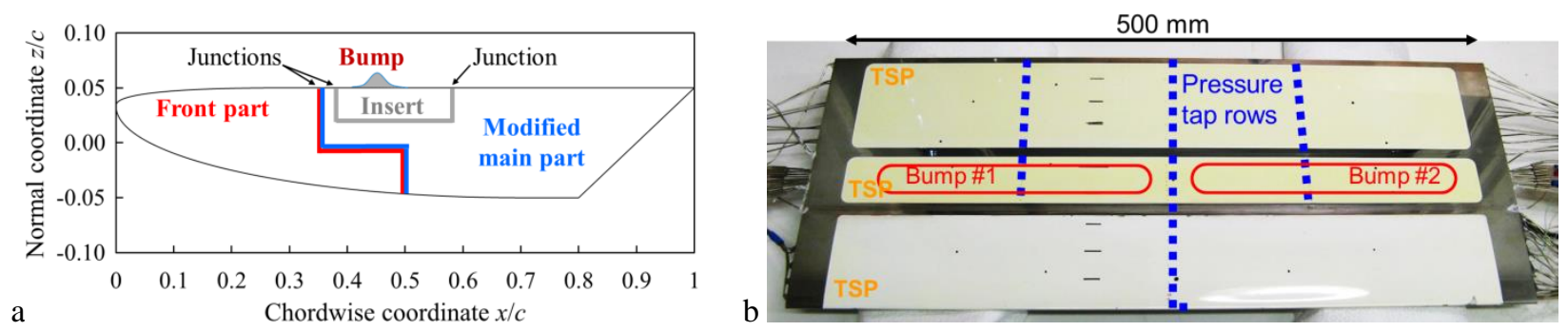

Figure 2: Simplified drawing of the BuLASTra model construction - side view (a); top view and instrumentation of the wind-tunnel model with the leading edge at the bottom of the image (b).

Three bumps with different nominal height $h=0.10 \mathrm{~mm}$ (small bump), $0.24 \mathrm{~mm}$ (mid bump) and $0.40 \mathrm{~mm}$ (big bump) were examined. They were manufactured on two different inserts: the mid bump was manufactured on the port half of one insert, which starboard half was left clean (i.e., bump-free) to serve as a reference surface; the small and big bumps were manufactured on the port and starboard halves of the other insert, respectively.

As can be seen from Figure 2, three junctions between the model parts were present on the model upper side. They were located at $x / c=35,37$ and $58 \%$. Small steps and gaps were observed at these junctions, but their size was compatible with laminar flow, since they fulfilled the criteria provided in Arnal (1992), Forte et al. (2015) and Costantini (2016). However, turbulent wedges were observed to arise from the two upstream junctions at large Reynolds numbers (see Sec. 4.4), probably originating from disturbances generated by micron-sized, three-dimensional roughness present at the junctions. (This very small roughness could be imperfections arising during the assembly of the model parts or particles transported by the flow and remained trapped in the aforementioned gaps.) The increase in the number of turbulent wedges at larger chord Reynolds numbers eventually limited the range of Reynolds numbers at which "natural" transition could be examined in the present work (see Sec. 3).

The model was equipped with a temperature-sensitive paint for global, non-intrusive transition detection (see Sec. 2.2.1) and with pressure taps to measure the surface pressure distribution (see Sec. 2.2.2). Oilfilm visualizations were also performed to visualize separated flow regions on the downstream side of the bump. The OFV setup is presented in Sec. 2.2.3.

\subsubsection{Temperature-Sensitive Paint (TSP) and TSP Data Analysis}

As shown in Figure 2b, the model was equipped with TSP, a global measurement technique discussed, e.g., in Liu and Sullivan (2005) and Tropea et al. (2007), and used in the present study for non-intrusive transition detection on the model upper side. Similarly to other thermographic methods, such as infrared thermography, boundary-layer transition is detected by measuring the temperature variation from the laminar to the turbulent regime. The measurement principle of TSP relies on temperature-sensitive, luminescent molecules, called luminophores. When excited by light in a specific wavelength range, the luminophores emit light in a different wavelength range, the intensity of which decreases at larger temperatures. In the present work, the luminophores were incorporated in a polyurethane-based coating, which was applied in pockets machined into the model surface, so that the final model contour did not present variations from design. The distribution of light emitted by the TSP was detected by means of 
Revision 1. Submitted to the Special Issue of Experimental Thermal and Fluid Science (ETFS) dedicated to the $5^{\text {th }}$ International Conference on Experimental Fluid Mechanics (ICEFM)

cameras (see below), thus enabling the global measurement of the temperature variation between the laminar and turbulent flow domains and therefore of boundary-layer transition. The TSP sensor was the same as that discussed in Ondrus et al. (2015). The model surface was polished to an average roughness of $R_{a}=0.037 \pm 0.011 \mu \mathrm{m}$ (mean roughness depth of $R_{z}=0.31 \pm 0.09 \mu \mathrm{m}$ ). These values are the average and standard deviation of the roughness measurements at 25 different locations on the TSP surface, which were conducted by means of a contact profilometer with a vertical resolution of $\pm 0.8 \mathrm{~nm}$ for a measurement length of $\Delta x=1.75 \mathrm{~mm}$. The final contour of the bumps was also measured by means of the aforementioned contact profilometer, in this case with a vertical resolution of $\pm 8 \mathrm{~nm}$ because of the larger size of the measurement domain: $\Delta x=24.5 \mathrm{~mm}$. This was the maximal measurement length supported by the used measurement device. An example of the final bump contour is shown in Figure 3a, where measurements of the mid-bump shape in the streamwise direction are presented for different spanwise locations, indicated in Figure 3b. The nominal bump contour is also shown in Figure 3a. As can be seen from this figure, the bump size and shape was essentially uniform in the spanwise direction. Moreover, the actual bump shape corresponded to the nominal contour. The average measured height of the bumps was slightly larger than the nominal one: $h=0.115 \mathrm{~mm}$ (small bump), $0.255 \mathrm{~mm}$ (mid bump) and $0.430 \mathrm{~mm}$ (big bump); the standard deviation from these average values was within $\Delta h= \pm 0.005 \mathrm{~mm}$. Because of the aforementioned limitation in the maximal measurement length supported by the used contact profilometer, the actual streamwise extent of the bumps could not be measured accurately. Therefore, the nominal value of $a=20 \mathrm{~mm}$ was taken as streamwise extent of the bumps. The corresponding bump height-to-length ratios were $h / a=0.006$ (small bump), 0.013 (mid bump) and 0.021 (big bump).
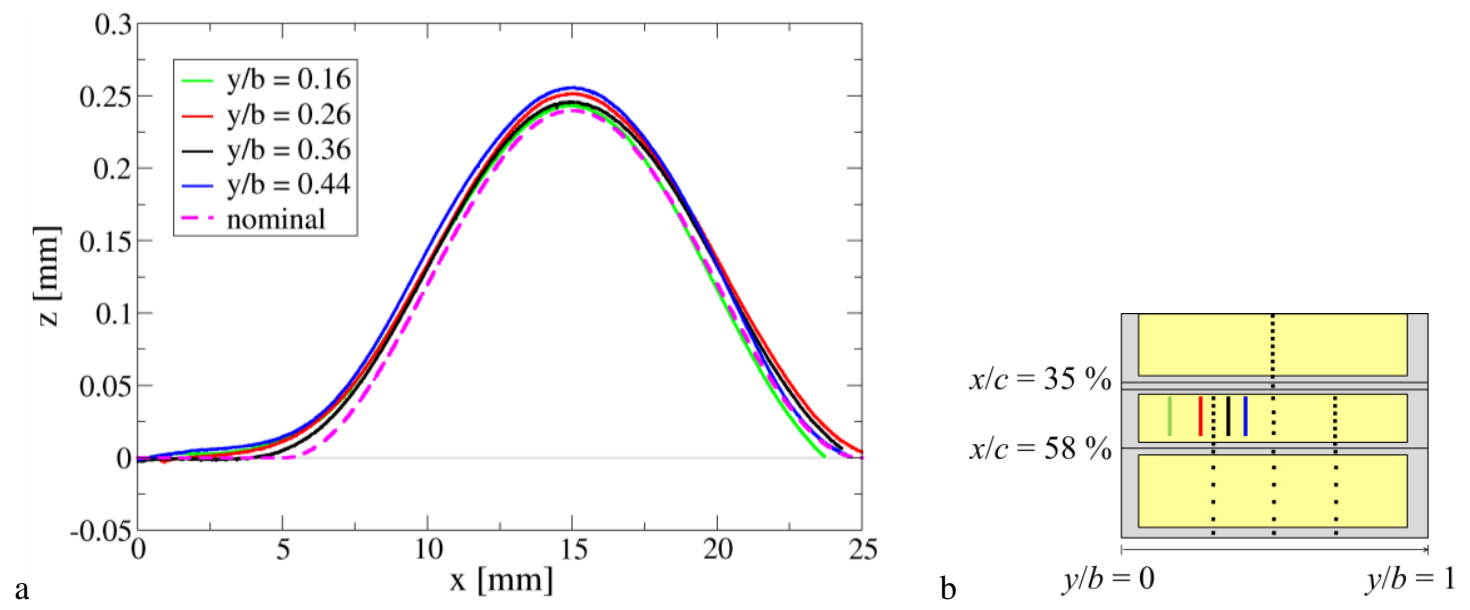

Figure 3: Measured contour of the mid bump, as compared to the nominal one (a), and indications of the measurement locations (b).

Two different optical setups for transition detection at DNW-KRG by means of TSP were used in this work: a standard setup, described in Costantini et al. (2016) and in Costantini (2016), and a more sophisticated setup, presented in Risius (2018). In both cases, the hardware had to be installed behind the side walls of the DNW-KRG test section, since optical access to the model from the adaptive top and bottom walls of the test section was not possible. Cameras and light sources were installed on both sides of the test section and were mounted in direct connection with the turntables, where the model was also fixed. In this manner, neither the distribution of the excitation light nor the image of the model surface observed by the cameras changed when the angle-of-attack of the model was varied. An image of the BuLASTra model installed in the DNW-KRG test section is shown in Figure 4. The lighting conditions of this picture allow to notice the big bump (and - although more hardly - even the small bump) on the insert mounted on the considered model configuration. 
Revision 1. Submitted to the Special Issue of Experimental Thermal and Fluid Science (ETFS) dedicated to the $5^{\text {th }}$ International Conference on Experimental Fluid Mechanics (ICEFM)

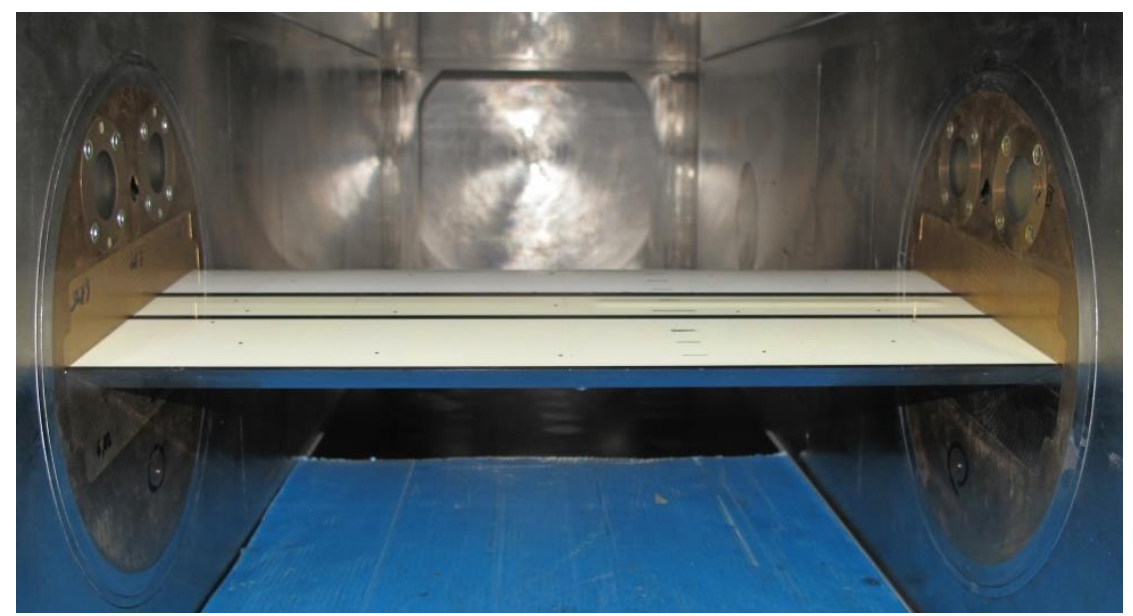

Figure 4: BuLASTra model mounted in the DNW-KRG test section, as seen from a downstream position.

The excitation light for the TSP was supplied by four high-power light emitting diodes (LEDs). Two LEDs were installed in each turntable behind suitable windows. In the case of the standard optical setup, these were circular windows, which are also visible in Figure 4. The specified excitation center wavelength of the LEDs was $\lambda=410 \mathrm{~nm}$; band-pass filters for the wavelength range 390-430 nm were placed in front of the LEDs in order to block light at smaller and larger wavelengths. The same type of LEDs was used for both optical setups.

The main differences between the two optical setups were the type of the cameras and their positioning. The cameras used for image acquisition with the standard optical setup were two miniaturized chargecoupled device $(C C D)$ cameras with 12-bit dynamic range and a $1392 \times 1024$ pixels sensor. Each camera was equipped with a pinhole lens (with a focal length of $4 \mathrm{~mm}$ ) and mounted directly at the turntable, with the lenses fitted through the turntables (see Costantini et al. (2016)). In the case of the more sophisticated optical setup, two complementary metal-oxide-semiconductor (CMOS) cameras were used, with 16-bit dynamic range and a $2560 \times 2160$ pixels sensor. The size of these cameras was significantly larger than that of the cameras used in the standard optical setup. The CMOS cameras could not be mounted directly at the turntables, but were connected to the turntables via a metallic frame. The CMOS camera axes were oriented in a direction nearly perpendicular to the test-section side walls, and images of the model upper surface were captured via a "periscope", i.e., using two mirrors oriented at appropriate angles (see Risius (2018)). The lenses had a focal length of $28 \mathrm{~mm}$, and a Scheimpflug adapter was mounted between lens and camera sensor to achieve a suitable depth-of-focus. In both optical setups, high-pass spectral filters with a cutoff wavelength of $\lambda=590 \mathrm{~nm}$ were installed between lenses and camera sensors to block light at smaller wavelengths, while at the same time allowing transmittance of the light emitted by the TSP. The image acquisition frequency was markedly larger with the "periscope" setup: $100 \mathrm{~Hz}$, instead of $5 \mathrm{~Hz}$ with the standard optical setup. (The exposure time of the camera sensors were 7.5 and $15 \mathrm{~ms}$, respectively.) The higher acquisition frequency enabled to increase the number of TSP images within the data evaluation window (see Sec. 2.1) from 1 to 10. The averaging of these TSP images, coupled with the larger signal-to-noise ratio of the CMOS cameras, led to a significant increase in the signal-to-noise ratio of the TSP results, as compared to that achieved with the standard optical setup. This will be seen in some of the series of TSP results presented in Sec. 4. Nevertheless, the processing of the acquired TSP images was the same for both optical setups. The TSP data acquisition was managed automatically using the inhouse software package ToPas, which was presented in Klein et al. (2005). All image operations were also conducted using ToPas. As described in Costantini et al. (2016) and Costantini (2016), reference TSP images were acquired before the actual run in DNW-KRG had started. These reference images were averaged and then divided by the (average) TSP image obtained in the data evaluation window ("run" image). (Before division, reference and run images were aligned to compensate for the small image deformation due to aerodynamic loading.) The divided TSP image was finally projected (mapped) onto a 
Revision 1. Submitted to the Special Issue of Experimental Thermal and Fluid Science (ETFS) dedicated to the $5^{\text {th }}$ International Conference on Experimental Fluid Mechanics (ICEFM)

three-dimensional grid representing the model upper surface. This procedure was based on the circular markers applied on the TSP surface, which are visible in Figure $2 b$ and in Figure 4. The final TSP results, obtained after merging of the mapped TSP images acquired by the two cameras, will be shown as they would be seen from the inaccessible top wall of the test section. Regions with too small signal-to-noise ratio were masked white. These regions clearly included also the strips of width $\Delta(x / c)=6 \%$ around the junctions, which were left uncoated (see Figure $2 b$ and Figure 4 ) to guarantee a high surface quality of the interfaces between the model parts.

The transition location was defined in the present work as the location corresponding to the maximal gradient of the surface temperature within the transitional region. This choice was motivated by the coincidence found in Kreplin and Höhler (1992), Ashill et al. (1996) and Lemarechal et al. (2018) between this point and that corresponding to the maximal value of the root-mean-square of hot-film signals within the transitional region. The transition location was determined in a manner analogous to that discussed in Costantini et al. (2016) and Costantini (2016); in the present work, however, one transition location was obtained for each bump configuration. Streamwise distributions of the TSP normalized intensity were evaluated on the final TSP results at five spanwise sections for each model half. The spanwise locations of the evaluation sections were maintained unvaried for all examined test conditions. The evaluation sections were in regions sufficiently distant from the turbulent wedges arising from the pressure taps and from surface contamination, except for the big-bump configuration at the largest examined Reynolds number, which was affected by a large number of turbulent wedges (see Sec. 4.4). In order to increase the signalto-noise ratio, the intensity values were averaged over a spanwise range of $\Delta(y / b)= \pm 0.5 \%$ across each spanwise section. The TSP intensity distributions were then analyzed by means of an algorithm capable of detecting the maximal value of the intensity gradient in the transitional region (see Costantini (2016) for more details). The transition locations $x_{\mathrm{T}} / c$ reported in this work are the averages of the transition locations evaluated at the five spanwise sections for each bump configuration. The uncertainty in $x_{\mathrm{T}} / c$ was evaluated as the standard deviation of the transition locations obtained at the five sections. The use of a single, welldefined, accurately and consistently measurable transition location was of fundamental importance in this work, where the effects of changes in various parameters on boundary-layer transition were systematically investigated.

\subsubsection{Pressure Taps}

The BuLASTra model was also instrumented with 90 pressure taps for the measurement of the surface pressure distributions. As shown in Figure $2 b$, the pressure taps were distributed in three rows aligned with the freestream direction. The main row of 54 pressure taps was placed at the model mid-span section $(y / b=0.5)$. Two additional pressure taps in the leading-edge region were installed at $y / b=0.48$ because of manufacturing constraints. The pressure taps in the mid-span region included also pressure taps on the model lower side and provided a reference pressure distribution in the bump-free area. The other two pressure tap rows were installed at $y / b=0.3$ and 0.7 ; each row consisted of 17 pressure taps. The distance between the pressure taps in the inserts was smaller than on the model main part and enabled to capture the bump-induced pressure variations. The locations of the pressure taps in the inserts were: $x / c=37.5$, $39,41,43,45,46.5,48,50,53$ and $57 \%$. The pressure taps at $x / c=39$ and $41 \%$ were placed upstream and downstream of the bump start, respectively, while the locations of the pressure taps at 45 and $50 \%$ corresponded to the locations of the bump crest and end. The orifices of the pressure taps had a diameter of $0.25 \mathrm{~mm}$. The circular shape of the orifices, the tap diameter and the sharpness of the orifice edges were ensured for all pressure taps, including those embedded in the TSP coating. This was accomplished via additional treatment of the orifices after TSP application, as described in Klein et al. (2015).

\subsubsection{Oil-Film Visualizations (OFV)}

Oil-film visualization is a technique applied in wind-tunnel testing since several decades (see, e.g., Merzkirch (1987) for an overview on OFV, and references therein for a review of previous applications). In the present work, OFV were carried out for a limited number of test conditions to verify the conjectures 
Revision 1. Submitted to the Special Issue of Experimental Thermal and Fluid Science (ETFS) dedicated to the $5^{\text {th }}$ International Conference on Experimental Fluid Mechanics (ICEFM)

on the presence or absence of separated flow regions on the downstream side of the bumps. In contrast to oil-film methods based on luminescent oils, such as those presented in Lee et al. (2018), a transparent silicone oil mixed with a black powder (black toner for laser printers) was used in the present work. The surface illumination was provided by the TSP itself, i.e., by the luminescence of the TSP surface excited by the LEDs. The optical setup used for TSP (see Sec. 2.2.1) could therefore be used also for OFV. The standard optical setup was used for the oil-film visualizations, but in this case the images were acquired at the maximal frequency of $10 \mathrm{~Hz}$ achievable with the CCD cameras used in this setup. (The CCD exposure time was reduced to $8 \mathrm{~ms}$.) In the OFV images, darker areas correspond to regions where the dyed oil has accumulated via the action of the flow, while brighter areas correspond to regions where the dyed oil has been transported away. Note that the used OFV setup allowed to avoid any modification of the model contour, such as that due to the use of adhesive foils, which would have been particularly critical in the bump regions.

As discussed in Schülein (2004), oil viscosity and oil-film application procedure have to be compatible with the short testing time in Ludwieg tubes and adequate to the examined test conditions. In the present experiments in DNW-KRG, silicone oils with a kinematic viscosity of approximately $5 \cdot 10^{-6} \mathrm{~m}^{2} / \mathrm{s}$ were found to reach steady conditions in the case of the big-bump configuration and were used to visualize separated flow regions on this model configuration (see Sec. 4.1). The dyed oil (mixture of oil and black powder) was applied on the model surface by means of a sponge. A typical OFV image acquired before a run had started is shown in Figure 5. This image was obtained after application of the same image processing procedure presented in Sec. 2.2.1 for the TSP images: the OFV images acquired by the two cameras were also mapped onto the three-dimensional grid representing the model surface and merged to obtain the final OFV image shown in Figure 5a. Regions with too little signal were masked white. A detail of the region indicated by the orange rectangle in Figure 5a is presented in Figure 5b. The red, blue and green lines show the locations corresponding to $x / c=40,45$ and $50 \%$, i.e., to the start, crest and end of the bumps. It should be noted here that the tick markers visible in Figure 5b, which were aimed to indicate the locations corresponding to $x / c=40$ and $50 \%$, were erroneously applied on the TSP surface of the insert at more downstream locations. The error was as large as $\Delta(x / c)=2.5 \%$ (in the case of the tick marker at the location that should correspond to $x / c=50 \%$ ) and could be revealed only after accurate mapping of the TSP and OFV images. This observation is a further confirmation of the importance of analyzing mapped data to obtain accurate measurements, instead of relying on tick markers applied on the model surface. The red dots in Figure $5 \mathrm{~b}$ show the locations of the pressure taps in the considered region (see Sec. 2.2.2). The match between the mapped positions of the pressure taps and their actual locations was excellent. Note here that the pressure taps were not blocked by the oil during the short testing time in DNW-KRG, probably because of the turbulent wedges spreading over the pressure tap rows, which rapidly transported the oil film away from the pressure taps. Moreover, neither the pressure tap tubings nor the TSP were damaged by the oil film, even though it was left on the model surface for up to three days before removing it.

At this point of the description, it should be emphasized that the oil-film visualizations were timeconsuming and had very high wind-tunnel operation costs per data point. For this reason, the tests with OFV had to focus on only one model configuration. The configuration with two bumps (small and big) was selected for this study. Some runs were also dedicated to the configuration with the mid bump, but the OFV were not conclusive with regard to the presence / absence of a separated flow region on the downstream side of this bump. The size of a laminar separation bubble, if present, is expected to be small, and both the oil viscosity and the oil-film application procedure should be optimized for this specific investigation. 

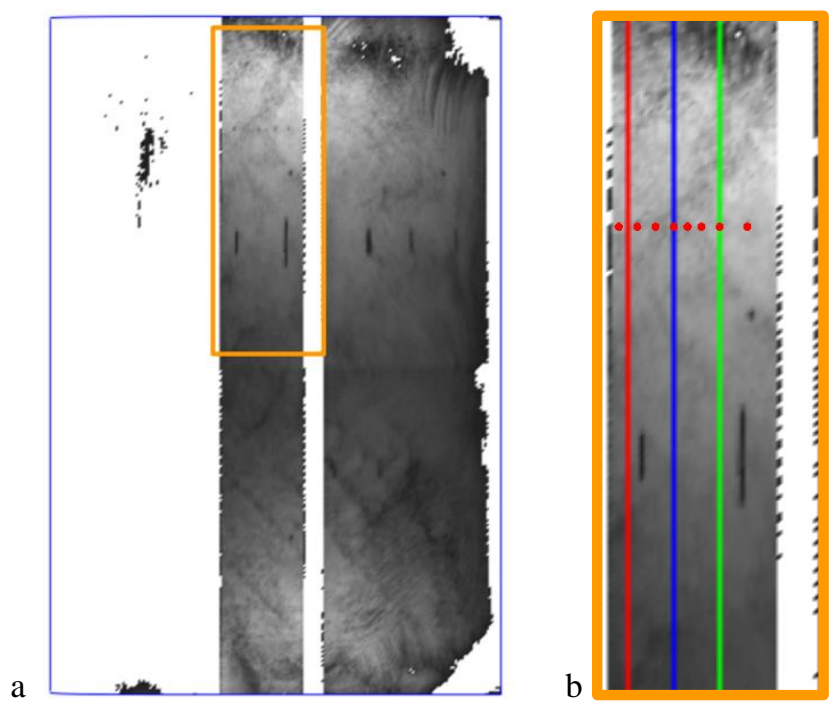

Figure 5: Final (mapped) image of the model upper surface with applied oil film before start of a run. a: whole model surface; b: detail of the area indicated by the orange rectangle. The red, blue and green lines show the locations corresponding to $x / c=40,45$ and $50 \%$, respectively, while the red dots show the locations of the pressure taps.

\section{Test Parameters and Experimental Conditions}

The experiments were conducted in a (quasi-) two-dimensional flow at freestream Mach numbers from $\mathrm{M}=U_{\infty} / a_{\infty}=0.35$ to 0.77 and chord Reynolds numbers from $\operatorname{Re}=U_{\infty} c / v_{\infty}=3.5$ to $10 \cdot 10^{6}$, where $U_{\infty}$, $a_{\infty}$ and $v_{\infty}$ are the velocity, the speed of sound and the kinematic viscosity of the freestream. The examined test conditions are summarized in Table 1. The maximal chord Reynolds number that could be investigated was limited by the number of turbulent wedges observed on the model surface at the largest Reynolds numbers: a detection of "natural" transition was not possible at chord Reynolds numbers larger than those reported in Table 1.

Table 1: Examined test conditions.

\begin{tabular}{|c|c|c|}
\hline $\mathbf{M}$ & $\boldsymbol{\operatorname { R e }}\left[\mathbf{1 0}^{\mathbf{6}}\right]$ & $\boldsymbol{\beta}_{\mathbf{H}}$ \\
\hline 0.35 & $3.5,6,8$ & -0.012 to 0.080 \\
\hline 0.50 & $4.5,6,8$ & 0.015 to 0.117 \\
\hline 0.65 & 6 & 0.061 \\
\hline 0.77 & $6,8,10$ & 0.063 to 0.112 \\
\hline
\end{tabular}

The bump height-to-length ratio $h / a$ (see Sec. 2.2.1) was chosen as the characteristic parameter for the imperfection size. This enabled to compare the present results with those obtained in previous work, where $h / a$ was also the characteristic parameter used to quantify the effect of bumps on boundary-layer transition. Note, however, that only the bump height $h$ was varied in the present work, while the bump length $a$ was kept fixed.

Various streamwise pressure gradients relevant for NLF surfaces were implemented in the present work. In most cases, favorable pressure gradients were considered, since these are the most relevant for NLF surfaces. Nevertheless, at the two lower Mach numbers also nearly-zero pressure gradients were examined, and a weak adverse pressure gradient was additionally considered at $M=0.35$. The Hartree parameter $\beta_{\mathrm{H}}$ of the self-similar solution of the boundary-layer equations (Falkner-Skan equation) was selected as the characteristic parameter for the global pressure distribution, as suggested, e.g., in Meyer and Kleiser (1989). The value of the Hartree parameter was determined for the smooth (i.e., bump-free) surface in the same manner as that presented in Costantini et al. (2016) and Costantini (2016). The value of the Hartree parameter was evaluated as the average value over that chordwise region where the pressure 
Revision 1. Submitted to the Special Issue of Experimental Thermal and Fluid Science (ETFS) dedicated to the $5^{\text {th }}$ International Conference on Experimental Fluid Mechanics (ICEFM)

gradient had been essentially uniform. This region was approximately $20 \% \leq x / c \leq 70 \%$ for the cases with nearly-zero $\left(\beta_{\mathrm{H}} \sim 0\right)$ and favorable pressure gradients $\left(\beta_{\mathrm{H}}>0\right)$, whereas it was approximately $5 \% \leq x / c \leq 40 \%$ for the case with an adverse pressure gradient $\left(\beta_{\mathrm{H}}<0\right)$.

It should be remarked here that, by virtue of the working principle of the DNW-KRG Ludwieg-tube facility (see Koch (2004) and Costantini et al. (2016)), the ratio between surface and adiabatic-wall temperatures $T_{w} / T_{a w}$ was larger than 1 during the test runs: it was in the range $T_{w} / T_{a w}=1.040-1.065$ at $\mathrm{M}=0.77$ and decreased to $T_{w} / T_{a w}=1.020-1.040$ at $\mathrm{M}=0.35$. The charge temperature of the gas in the DNW-KRG storage tube was set to approximately $T_{\mathrm{c}}=288 \mathrm{~K}$ for all test conditions, but the value of the freestream temperature during a run (and therefore also of $T_{a w}$ ) depended on the Mach number (see Rosemann (1997), Koch (2004), Costantini et al. (2016) and Costantini (2016)). The adiabatic-wall temperature was in the range $T_{a w}=268.5-272.5 \mathrm{~K}$ at $\mathrm{M}=0.77$ and increased to $T_{a w}=277.5-280.5 \mathrm{~K}$ at $\mathrm{M}=0.35$. The model surface temperature range was $T_{w}=284.5-287.5 \mathrm{~K}$. (The temperatures $T_{w}$ and $T_{a w}$ were evaluated at $x / c=15 \%$, where the boundary layer was laminar at all examined test conditions.) As discussed in Arnal (1992), Schlichting and Gersten (2000), Costantini et al. (2016), Costantini (2016) and Costantini et al. (2018), a non-adiabatic surface has an effect on boundary-layer stability and transition in flows over smooth surfaces. In Costantini (2016) and Costantini et al. (2018), a non-adiabatic surface was also found to have an influence on transition induced by forward-facing steps, at least up to a certain value of the non-dimensional step parameters. In Al-Maaitah et al. (1990), a variation in the wall temperature ratio $T_{w} / T_{a w}$ was shown to have an impact on boundary-layer stability even in the presence of a bump. Analogously to its influence on step-induced transition, a non-adiabatic surface is expected to have an influence also on bump-induced transition, at least up to a certain bump height-to-length ratio. The experimental investigation of this effect in DNW-KRG is, however, very time-consuming, and therefore associated with high wind-tunnel operation costs per data point (see Costantini (2016)). An investigation of the influence of a non-adiabatic surface on bump-induced transition could not be carried out in the present study, but is planned for future work.

Details on the evaluation of the aforementioned parameters and on their uncertainties are given in Costantini et al. (2016) and Costantini (2016).

\section{Results}

The experimental results are presented in different sections, where the effect on boundary-layer transition of the variation of one single parameter will be shown. The results were verified to be well repeatable and reproducible. In particular, the differences in the data (transition location included) obtained with the same model configurations in different wind-tunnel entries (separated by three to four months) were shown to be within the measurement uncertainties. Moreover, the transition locations measured with the smooth (bump-free) surface were in agreement with those measured in Costantini (2016) for the same test conditions on another wind-tunnel model with the same reference contour.

\subsection{Effect of the Bump Height-to-Length Ratio}

The effect of a larger bump height-to-length ratio on boundary-layer transition is shown in Figure 6 with the TSP results for a case at $\mathrm{M}=0.77, \mathrm{Re}=6 \cdot 10^{6}$ and a favorable global pressure gradient $\left(\beta_{\mathrm{H}}=0.096\right)$. Bright and dark regions correspond to areas of low and high wall heat flux, respectively, which is related to low and high wall shear stress; the detected locations of boundary-layer transition (see Sec. 2.2.1) are indicated in the TSP results by yellow dashed lines. The pressure tap rows caused up to three turbulent wedges; the other turbulent wedges arose from contamination of the model surface during wind tunnel operation. In spite of the turbulent wedges, natural transition was clearly shown to occur at a more upstream location $x_{\mathrm{T}} / c$ with increasing bump height-to-length ratio $h / a-$ see Figure 6 . This result is in agreement with findings from previous experimental (Fage (1943), Carmichael (1959), Perraud et al. (2004)) and numerical work (Nayfeh et al. (1988), Cebeci and Egan (1989), Masad and Iyer (1993), 
Revision 1. Submitted to the Special Issue of Experimental Thermal and Fluid Science (ETFS) dedicated to the $5^{\text {th }}$ International Conference on Experimental Fluid Mechanics (ICEFM)

Perraud et al. (2004)). The effect of larger bump height-to-length ratios on boundary-layer transition at all other test conditions examined in the present work was found to be similar to that discussed above.
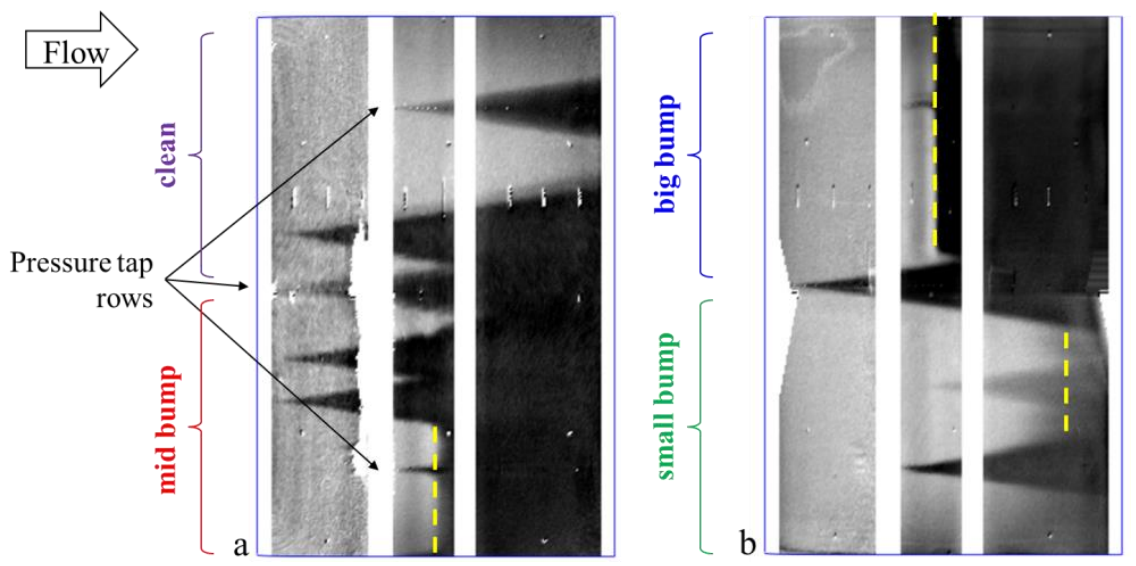

Figure 6: TSP results for different bump height-to-length ratios $h / a$ obtained at $\mathrm{M}=0.77, \operatorname{Re}=6 \cdot 10^{6}$ and $\beta_{\mathrm{H}}=0.096$. a: clean configuration (bump-free): fully laminar; small bump $(h / a=0.006): x_{\mathrm{T}} / c=82.6 \pm 2.0 \%$. b: mid

bump ( $h / a=0.013): x_{\mathrm{T}} / c=51.6 \pm 1.5 \%$; big bump $(h / a=0.021): x_{\mathrm{T}} / c=48.7 \pm 0.2 \%$. (The difference in the TSP signal-to-noise ratio of the two results is due to the different optical setups used in the experiments - see Sec. 2.2.1).

The surface pressure distributions measured with the two model configurations at the test conditions of Figure 6 are presented in Figure 7. The curves named "Reference (1)" and "Reference (2)" show the pressure distributions measured by means of the mid-span pressure tap row with the model configurations of Figure 6a and Figure 6b, respectively. All six pressure distributions (three pressure tap rows for each of the two model configurations) are in excellent agreement for most of the chord length, except for the region around the bumps. A zoomed-in plot of the bump region is shown in Figure $7 \mathrm{~b}$. The presence of the bumps led to significant differences in the pressure distributions, as compared to those obtained with the reference / clean surfaces; the magnitude of these differences clearly increased with increasing bump height-to-length ratio $h / a$. (The small deviations of the pressure distributions from ideally smooth ones, visible for the reference / clean surfaces, were due to small model contour variations remaining after treatment of the TSP surface.) With respect to its development on the reference / clean surfaces, the boundary layer was first slightly decelerated when it approached the bumps, then accelerated on the upstream side of the bumps and decelerated on their downstream sides, until the global pressure distribution was finally recovered at a certain distance downstream of the bump end. The exact location of the recovery of the reference / clean surface pressure distribution appears to be more downstream with increasing bump height-to-length ratio. Note that the influence of the local flow acceleration / deceleration over the bumps can be seen also in the TSP results of Figure 6. In fact, the (still laminar) accelerating boundary layer over the upstream side of the bumps was characterized by a higher wall shear stress, and therefore higher wall heat flux, than that of the boundary layer immediately upstream of the start of the bump, resulting in a darker strip in the TSP data at $x / c=40-45 \%$. Similar, but opposite, considerations apply for the decelerating boundary layer over the downstream side of the bumps, where the wall shear stress (and the wall heat flux) was lower than that in the surrounding regions and led to a brighter strip in the TSP data at $x / c=45-48 \%$ (i.e., immediately downstream of the bump crest). It can be seen in Figure $7 \mathrm{~b}$ that the deceleration on the downstream side of the bumps was more pronounced than the acceleration on their upstream side; this is in agreement with findings from previous work (see Nayfeh et al. (1988) and Arnal (1992), among others) and eventually induced earlier transition (see Figure 6). 
Revision 1. Submitted to the Special Issue of Experimental Thermal and Fluid Science (ETFS) dedicated to the $5^{\text {th }}$ International Conference on Experimental Fluid Mechanics (ICEFM)
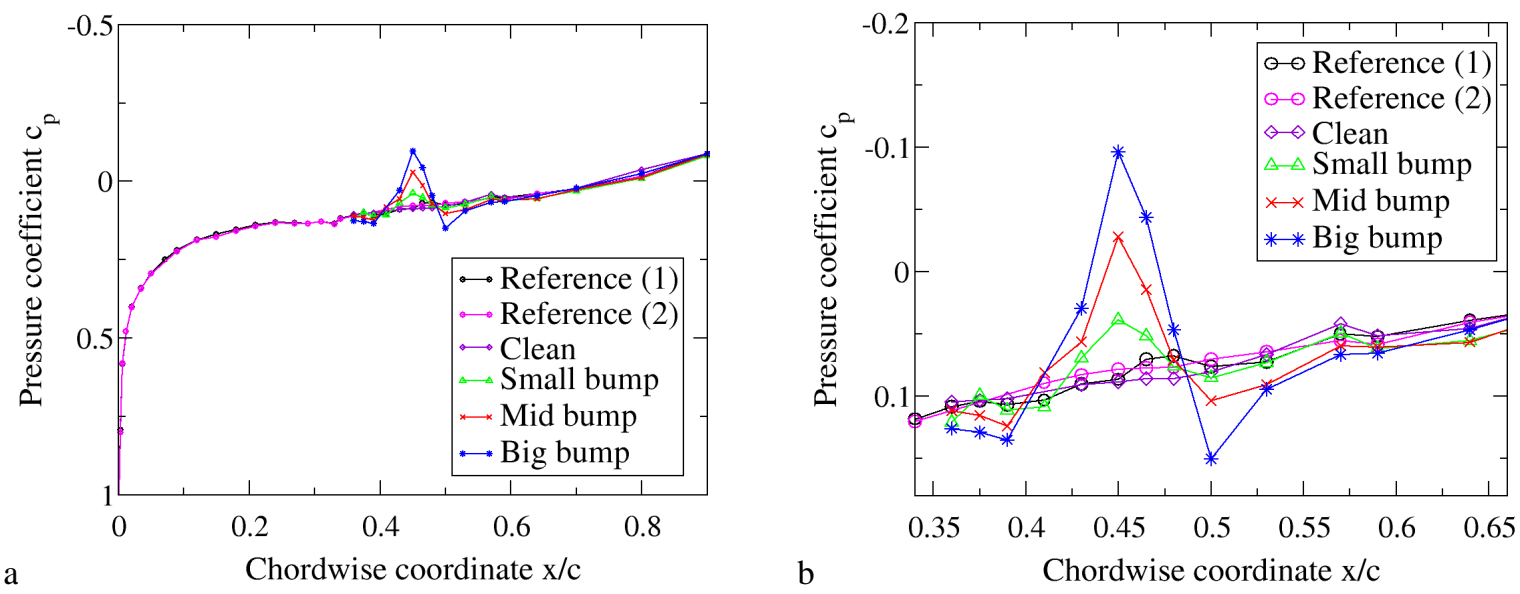

Figure 7: Surface pressure distributions measured for the case of Figure 6. a: over the whole chord length. b: zoomed-in around the bump region. Start and end of the bumps are located at $x / c=40 \%$ and $50 \%$, respectively.

As discussed, e.g., in Arnal (1992), Nayfeh et al. (1988), Cebeci and Egan (1989), Masad and Iyer (1993) and Franco et al. (2018), the adverse influence of surface bumps on boundary-layer stability and transition is particularly marked when the deceleration on the downstream side of the bump is so strong as to induce flow separation, since amplification of streamwise instabilities is enhanced in separated flow regions. In the present work, the boundary layer indeed separated in the presence of the big bump, as it will be discussed below. This led to transition occurring close to the bump crest in the case shown in Figure 6 and at all other examined test conditions. It should be remarked here that the influence of a laminar separation bubble on the surface pressure distributions over the big bump was not visible at $\operatorname{Re} \geq 6 \cdot 10^{6}$, such as in the case of Figure 7, since the surface pressure measurements were affected by a turbulent wedge spreading over the pressure tap row (see Figure 6). An example of surface pressure distribution measured at $\operatorname{Re}<6 \cdot 10^{6}$ in the absence of turbulent wedges is presented below (Figure 10). In any case, the presence of a laminar separation bubble (with turbulent reattachment) on the downstream side of the big bump was verified for different global pressure gradients via oil-film visualizations, which were performed for the lowest chord Reynolds numbers examined at $\mathrm{M}=0.50,0.65$ and 0.77 (see Sec. 2.2.3). An example series of oil-film images acquired at $\mathrm{M}=0.50, \mathrm{Re}=4.5 \cdot 10^{6}$ and $\beta_{\mathrm{H}}=0.095$ are shown in Figure 8a-e. The displayed area corresponds to that highlighted by the orange rectangle in Figure 5 . The oil-film images were taken at different time instants after the fast-acting valve of DNW-KRG had been opened. In particular, Figure $8 \mathrm{a}$ and Figure 8e were acquired just after the start and the end of the run, respectively, while Figure 8d was taken in the time window in which wind-tunnel and TSP data were evaluated (see Sec. 2.1). The corresponding TSP result for the same model surface region is shown in Figure 8f. Note that the presented oil-film images were filtered using a $1 \times 11(x \times y)$ median spatial filter. 

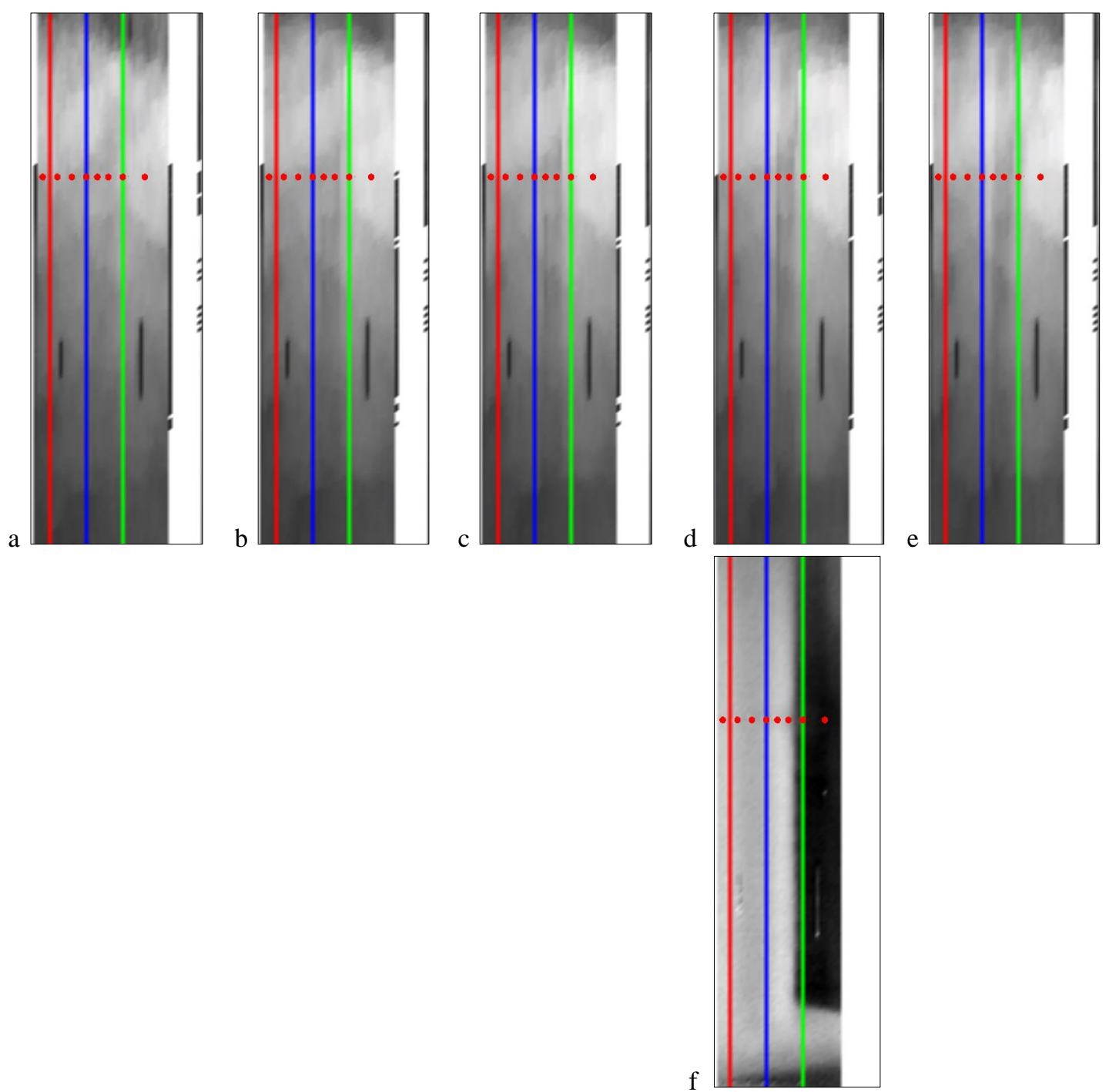

Figure 8: Oil-film visualizations of the big-bump region at $\mathrm{M}=0.50, \mathrm{Re}=4.5 \cdot 10^{6}$ and $\beta_{\mathrm{H}}=0.095$. a: just after opening of the DNW-KRG fast-acting valve; b: $0.3 \mathrm{~s}$ after image a; c: $0.1 \mathrm{~s}$ after image b; d: $0.2 \mathrm{~s}$ after image c; e: end of the run $\left(0.1 \mathrm{~s}\right.$ after image d). f: TSP result $\left(x_{\mathrm{T}} / c=49.7 \pm 0.1 \%\right)$.

After the run had started, the oil film was accelerated up to the bump crest, and especially over the upstream side of the bump (i.e., between the red and the blue lines in Figure 8). On the downstream side of the bump (i.e., between the blue and the green lines in Figure 8), an accumulation of the dyed oil can already be seen in Figure $8 \mathrm{~b}$ in the form of a darker strip between the blue and the green lines. This image was taken $0.3 \mathrm{~s}$ after the image shown in Figure 8a. The strip became more clearly visible in Figure $8 \mathrm{c}$ and Figure 8d, which were taken 0.4 and $0.6 \mathrm{~s}$ after the image shown in Figure 8a. In the time window between 0.3 and $0.7 \mathrm{~s}$, the strip was found to be quasi-steady, except for some local variations at different spanwise locations. In particular, a comparison of Figure $8 \mathrm{~b}$ and Figure 8c shows an upstream movement of the dyed oil in the region between the pressure tap row and the starboard end of the image. At the end of the run (Figure 8e), the darker strip was eventually smeared by the abrupt change in the flow conditions, which were no longer steady.

The darker strip in Figure 8b-d appears as the result of the accumulation of dyed oil due to the action of the boundary layer from both sides of the strip, and thus is likely to correspond to the "dead air" region within a laminar separation bubble with turbulent reattachment (see, e.g., Horton (1968), Kirk and Yarusevych (2017) and Miozzi et al. (2019)) occurring on the downstream side of the bump. A schematic 
of the structure of a time- and span-averaged laminar separation bubble (with turbulent reattachment) on the downstream side of the bump is shown in Figure 9a. In this schematic, the laminar separation bubble is ideally subdivided into two main regions: the dead-air region, where the reverse flow is weak, and a region of more intense reverse flow, located downstream of the dead-air region and terminating at the reattachment point. The corresponding streamwise distribution of the skin-friction coefficient is sketched in Figure 9b. In the dead-air region, the value of the skin-friction coefficient is negative but relatively low, while it decreases markedly in the region of more intense reverse flow. The reason for the enhancement in the reverse flow intensity within the separation bubble is the onset of transition in the shear layer, which approximately marks also the end of the dead-air region. As discussed in Sec. 2.2.1, the transition location was defined in the present work as the location corresponding to the maximal gradient of the surface temperature, and is therefore expected to be downstream of the end of the dead-air region, as illustrated in Figure 9.

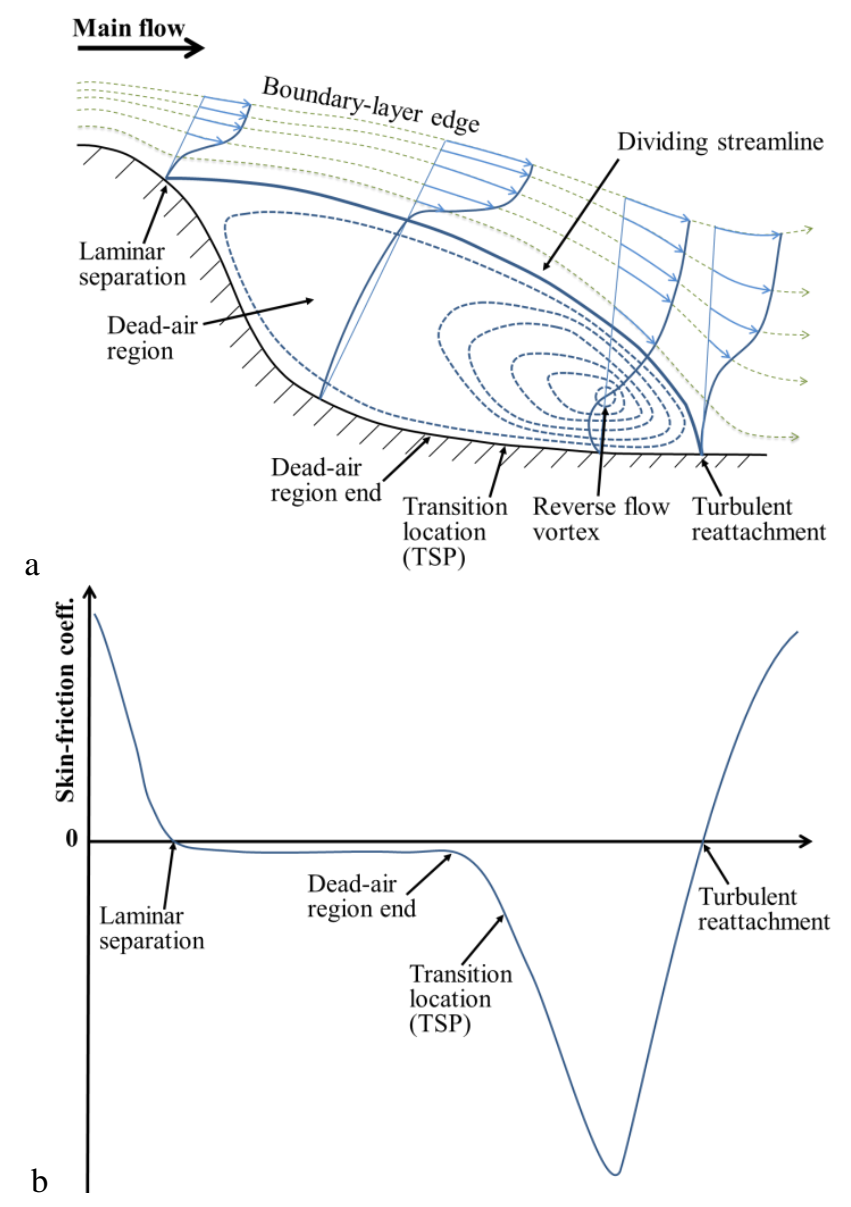

Figure 9: Schematic of the structure of a time- and span-averaged laminar separation bubble on the downstream side of a bump (a) and corresponding streamwise distribution of the skin-friction coefficient (b). The schematics are adapted from Horton (1968) and Selig et al. (2011), respectively.

In the OFV of Figure 8a-e, upstream of the darker strip, the oil film was transported (via the action of the wall shear stress) in the streamwise direction towards the dead-air region, where it accumulated. Analogously, but in the direction opposite to that of the freestream, the dyed oil was transported towards the dead-air region also from downstream of the strip; this was due to the action of the (negative) wall shear stress in the region of intense reverse flow (see Figure 9b). In a manner similar to that discussed in 
Revision 1. Submitted to the Special Issue of Experimental Thermal and Fluid Science (ETFS) dedicated to the $5^{\text {th }}$ International Conference on Experimental Fluid Mechanics (ICEFM)

Selig et al. (2011), the dyed oil accumulated in the region where the weakening skin-friction force and the surface tension (or oil adhesion) balanced. At this point of the discussion, it should be emphasized that the above description is a strong simplification of the complex flow dynamics within laminar separation bubbles, which generally show significant variations in space and time even for a two-dimensional steady freestream, as discussed by Kirk and Yarusevych (2017) and Miozzi et al. (2019), among others. In particular, the schematics shown in Figure 9 are presented only for illustrative purposes, without any intention to quantitatively describe the flow development in the presence of a laminar separation bubble with turbulent reattachment. Moreover, the images presented in Figure 8a-e were averaged over the CCD exposure time of $8 \mathrm{~ms}$. Nevertheless, from the above considerations, it can be reasonably assumed that the upstream side of the darker strip corresponded to the separation line of a laminar separation bubble at least to a first approximation and in a time-averaged sense. Similarly, the downstream side of the darker strip can be assumed to be located at the end of the dead-air region, slightly upstream of the transition location (as defined in this work, see Sec. 2.2.1), and certainly upstream of the turbulent reattachment location. The accumulation of dyed oil in a region upstream of the reattachment location has been discussed in more detail in Selig et al. (2011).

The interpretation of the oil-film images presented above is substantiated by a comparison of the chordwise distribution of the luminescent intensity of Figure 8d with the corresponding TSP intensity (from Figure 8f) and surface pressure distributions. This comparison is shown in Figure 10, where the red and green lines correspond to the chordwise intensity distributions extracted from the TSP result and from the oil-film image, respectively, at the spanwise location $y / b=0.71$ (i.e., at a distance of $\Delta(y / b)=0.01$ from the pressure tap row). The TSP intensity distributions were normalized in a manner such that low and high normalized intensities correspond to low and high wall shear stress, respectively, whereas the intensity distributions of the oil-film image were normalized with respect to the minimal and maximal intensity values in the region of the big bump. The surface pressure distributions measured in the mid-span section ("Reference") and in the bump region are shown in Figure 10 by black and blue symbols, respectively. The pressure data were measured simultaneously to the TSP result of Figure 8f.

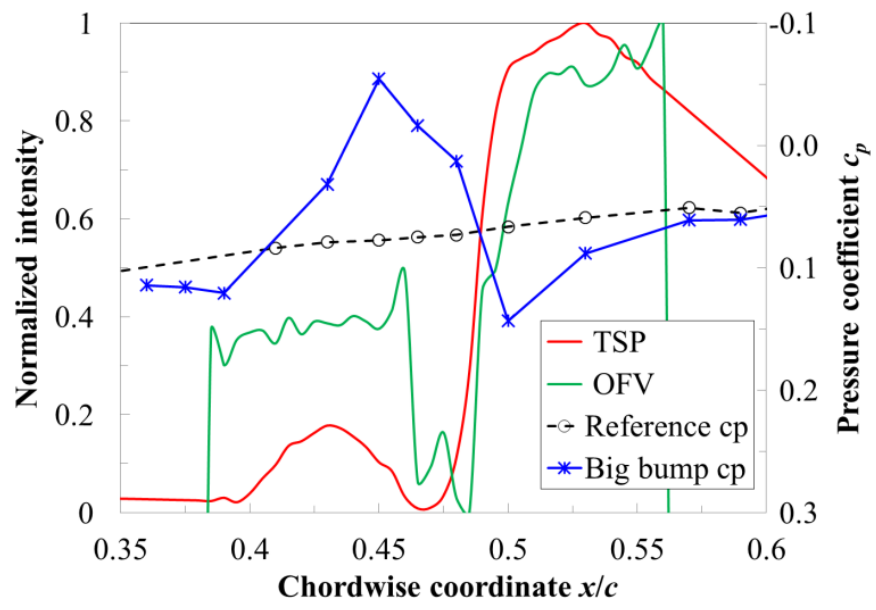

Figure 10: Comparison of surface pressure distributions and normalized intensities of TSP and oil film in the bigbump region for the case of Figure 8.

A comparison of TSP and oil-film intensity distributions shows that the first abrupt decrease in the oilfilm normalized intensity (corresponding to the upstream side of the aforementioned darker strip) occurred between $x / c=46$ and $46.5 \%$, i.e., slightly upstream of the minimum in the TSP normalized intensity distribution, which was found at $x / c=47 \%$. Further downstream, the marked increase in the oil-film normalized intensity (corresponding to the downstream side of the darker strip) started at $x / c=48.5 \%$, i.e., slightly upstream of the transition location, which was detected in the TSP result at $x_{\mathrm{T}} / c=49.7 \pm 0.1 \%$. Here, it should be emphasized again that the transition location was defined in the 
Revision 1. Submitted to the Special Issue of Experimental Thermal and Fluid Science (ETFS) dedicated to the $5^{\text {th }}$ International Conference on Experimental Fluid Mechanics (ICEFM)

present work as the location corresponding to the maximal gradient in the TSP intensity distribution, while the transition process in the laminar separation bubble had likely started at a more upstream location. Note also that the spatial resolution of the pressure tap row was not sufficient to adequately capture the pressure distribution induced by the presence of a laminar separation bubble, which influence would be otherwise visible as a pressure plateau downstream of the separation location, followed by a strong pressure recovery up to the turbulent reattachment location (see, for example, Kirk and Yarusevych (2017)). In (average) surface pressure distributions, the start of the transition process is typically detected at the location corresponding to the kink between the pressure plateau and the pressure recovery regions. Although the number and the locations of the pressure taps did not enable to capture adequately this characteristic pressure distribution, the start of the pressure plateau (i.e., the separation location), the start of the strong pressure recovery (i.e., the location of transition onset) and the end of the pressure recovery region can be at least estimated from the measured pressure distribution; the estimated locations are in line with the locations of separation, dead-air region end and transition obtained from the analysis of the oilfilm image and of the TSP result. It should be reminded here that an estimation of the aforementioned characteristic locations from the surface pressure distribution of Figure 10 was possible only because, at this relatively small chord Reynolds number $\left(\operatorname{Re}=4.5 \cdot 10^{6}\right)$, the surface pressure measurements were not affected by a turbulent wedge arising from the upstream pressure taps. This was not the case for the surface pressure distributions presented in Figure 7, which were measured within turbulent wedges.

The findings discussed above for the case at $\mathrm{M}=0.50, \mathrm{Re}=4.5 \cdot 10^{6}$ and $\beta_{\mathrm{H}}=0.095$ were confirmed also at the other flow conditions investigated via OFV. The locations of laminar separation $\left(x_{\mathrm{s}} / c\right)$ and end of the dead-air region $\left(x_{2} / c\right)$, obtained from the analysis of the oil-film images, are given in Table 2 , where the transition locations $x_{\mathrm{T}} / c$ detected in the TSP results are also reported.

Table 2: Locations of laminar separation, end of dead-air region and transition obtained from the analysis of the oilfilm images and of the TSP results at different flow conditions (big-bump configuration).

\begin{tabular}{|c|c|c|c|c|c|}
\hline $\mathbf{M}$ & $\boldsymbol{R e}\left[\mathbf{1 0}^{\mathbf{6}}\right]$ & $\boldsymbol{\beta}_{\mathbf{H}}$ & $\boldsymbol{x}_{\mathbf{s}} / \boldsymbol{c}(\mathbf{O F V})$ & $\boldsymbol{x}_{2} / \boldsymbol{c}(\mathbf{O F V})$ & $\boldsymbol{x}_{\mathrm{T}} / \boldsymbol{c}$ (TSP) \\
\hline 0.77 & 6 & 0.063 & $45.5-46.5 \%$ & $47.5 \%$ & $47.7 \pm 0.5 \%$ \\
\hline 0.77 & 6 & 0.096 & $45.5-46.5 \%$ & $48.0 \%$ & $48.7 \pm 0.2 \%$ \\
\hline 0.65 & 6 & 0.061 & $46.0-46.5 \%$ & $48.0 \%$ & $48.5 \pm 0.5 \%$ \\
\hline 0.50 & 4.5 & 0.015 & $46.0-46.5 \%$ & $48.5 \%$ & $49.1 \pm 0.3 \%$ \\
\hline 0.50 & 4.5 & 0.060 & $46.0-46.5 \%$ & $48.5 \%$ & $49.6 \pm 0.1 \%$ \\
\hline 0.50 & 4.5 & 0.095 & $46.0-46.5 \%$ & $48.5 \%$ & $49.7 \pm 0.1 \%$ \\
\hline 0.50 & 4.5 & 0.117 & $46.0-46.5 \%$ & $48.5 \%$ & $49.7 \pm 0.1 \%$ \\
\hline
\end{tabular}

Although the given values of $x_{\mathrm{s}} / c$ and $x_{2} / c$ should be taken as indicative, it can be seen from this table that the locations of laminar separation and dead-air region end did not change significantly at the examined test conditions. Moreover, the location $x_{2} / c$ was slightly upstream of the detected transition location for all examined cases. Since the TSP results and the surface pressure distributions measured in the presence of the big bump at different Reynolds numbers were essentially in agreement (see Sec. 4.4), it is expected that the findings summarized in this section hold also for the larger Reynolds numbers investigated in this work. Note also that, as a further confirmation of the present findings, the height-to-length ratio $h / a=0.021$ of the big bump was above the values of $h / a$ corresponding to separation onset over the downstream side of the bumps studied in Nayfeh et al. (1988), Cebeci and Egan (1989), Al-Maaitah et al. (1990), Masad and Iyer (1993), Masad and Malik (1995) and Wörner et al. (2002). As discussed in Masad and Nayfeh (1992), Masad and Iyer (1993) and Masad and Malik (1995), the size of the separated flow region in both streamwise and wall-normal directions is expected to increase at larger Mach numbers. In particular, separation is expected to occur at a slightly more upstream location at larger Mach numbers, while reattachment is expected to move towards a more downstream location. This behavior could not be verified with the measurements performed in the present work, although a slight upstream shift of $x_{\mathrm{s}} / c$ can 
Revision 1. Submitted to the Special Issue of Experimental Thermal and Fluid Science (ETFS) dedicated to the $5^{\text {th }}$ International Conference on Experimental Fluid Mechanics (ICEFM)

be supposed from the data reported in Table 2. In any case, no conclusion on the reattachment location can be derived from the available data.

Separated flow regions were not seen in the OFV on the small-bump configuration. This finding is in line with the results of Nayfeh et al. (1988), Cebeci and Egan (1989), Masad and Nayfeh (1992) and Masad and Iyer (1993), where no separation was observed on the downstream side of the bump for $h / a \leq 0.008$. As mentioned in Sec. 2.2.3, OFV of the mid-bump configuration were not conclusive. An analysis of the data of Nayfeh et al. (1988), Cebeci and Egan (1989), Al-Maaitah et al. (1990), Masad and Nayfeh (1992) and Masad and Iyer (1993) shows that $h / a=0.013$ is in a range of bump height-to-length ratios at which the flow may separate on the downstream side of the bump. In the range $0.009 \leq h / a \leq 0.015$, separation can occur depending on the test conditions, and in particular on the bump shape and on the Mach number. It cannot be excluded that, in the present work, the boundary layer separated on the downstream side of the mid bump at the larger Mach numbers, while it remained attached at the smaller Mach numbers. This may have also contributed to the different behavior of transition induced by the mid bump observed for larger and smaller Mach numbers, which is presented in the next section and discussed in more detail in Sec. 5.1.

\subsection{Effect of the Global Pressure Gradient}

The influence of a variation in the global pressure gradient on transition in the presence of small and big bumps is shown in Figure 11, where TSP results obtained at $\mathrm{M}=0.77$ and $\mathrm{Re}=6 \cdot 10^{6}$ are presented. Three different global pressure gradients were investigated: $\beta_{\mathrm{H}}=0.063$ (Figure 11a), 0.076 (Figure 11b) and 0.096 (Figure 11c). As can be seen in this figure, an increase in the Hartree parameter (i.e., a more pronounced global flow acceleration) led to a significant movement of boundary-layer transition into a more downstream location even in the presence of a bump with a height-to-length ratio of $h / a=0.006$ (small bump): from $x_{\mathrm{T}} / c=61.9 \pm 0.1 \%$ at $\beta_{\mathrm{H}}=0.063$ to $x_{\mathrm{T}} / c=82.6 \pm 2.0 \%$ at $\beta_{\mathrm{H}}=0.096$. In contrast, a variation of the global pressure gradient was found to have no appreciable influence on boundary-layer transition in the presence of the big bump $(h / a=0.021)$; this was very likely due to the occurrence of transition over a laminar separation bubble induced by the marked adverse pressure on the downstream side of the big bump (see Sec. 4.1), which was not affected significantly by a change in the global pressure gradient. The pressure distributions measured for the cases of Figure 11 are presented in Figure 12, where the dashed lines show the distributions corresponding to the mid-span model section ("reference"), whereas the solid lines show the distributions measured in the presence of the bumps. Different colors correspond to the data obtained at different global pressure gradients. The pressure distributions measured in the presence of the small and big bumps are shown in Figure 12a and Figure 12b, respectively. Also in these cases, the pressure distributions measured with and without bumps were in excellent agreement, except for the regions around the bumps. Zoomed-in plots of these regions are shown in Figure 12c and Figure $12 \mathrm{~d}$ for the surfaces with the small and big bumps, respectively. As can be seen from these figures, larger global flow acceleration did not lead to appreciable differences in the local surface pressure variations induced by the bumps. 
Revision 1. Submitted to the Special Issue of Experimental Thermal and Fluid Science (ETFS) dedicated to the $5^{\text {th }}$ International Conference on Experimental Fluid Mechanics (ICEFM)
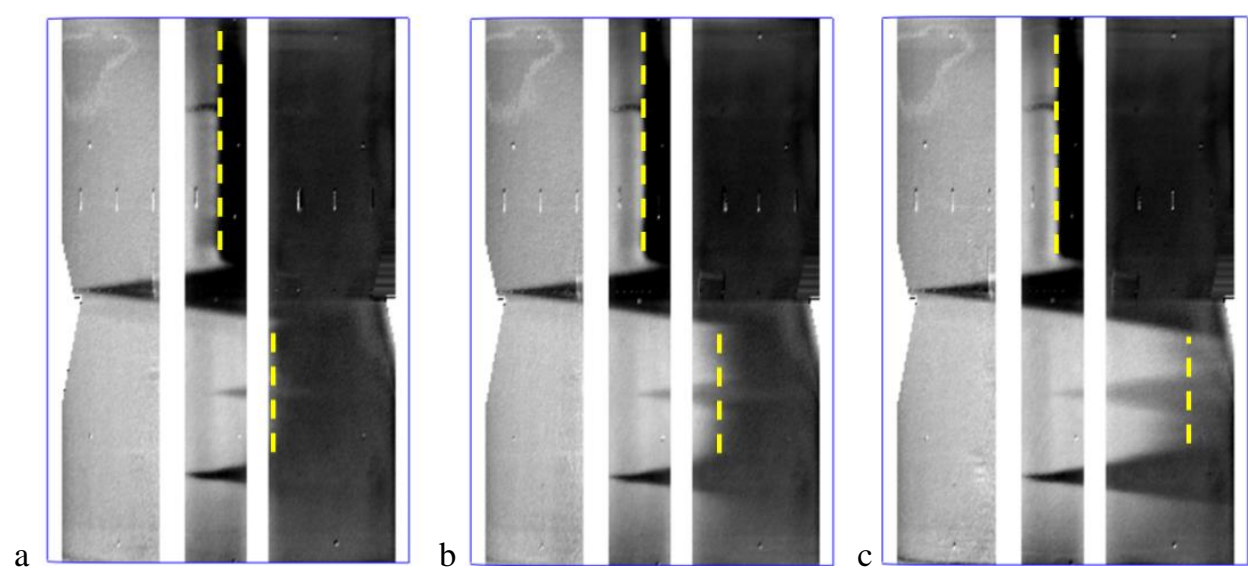

Figure 11: TSP results for different global pressure gradients $\beta_{\mathrm{H}}$ obtained with the small $(h / a=0.006)$ and big

$(h / a=0.021)$ bumps at $\mathrm{M}=0.77$ and $\operatorname{Re}=6 \cdot 10^{6} . \mathrm{a}: \beta_{\mathrm{H}}=0.063\left(x_{\mathrm{T}} / c=61.9 \pm 0.1 \%\right.$ vs. $\left.x_{\mathrm{T}} / c=47.7 \pm 0.5 \%\right)$; b: $\beta_{\mathrm{H}}=0.076\left(x_{\mathrm{T}} / c=66.7 \pm 1.2 \%\right.$ vs. $\left.x_{\mathrm{T}} / c=48.4 \pm 0.1 \%\right) ; \mathrm{c}: \beta_{\mathrm{H}}=0.096\left(x_{\mathrm{T}} / c=82.6 \pm 2.0 \%\right.$ vs. $\left.x_{\mathrm{T}} / c=48.7 \pm 0.2 \%\right)$.

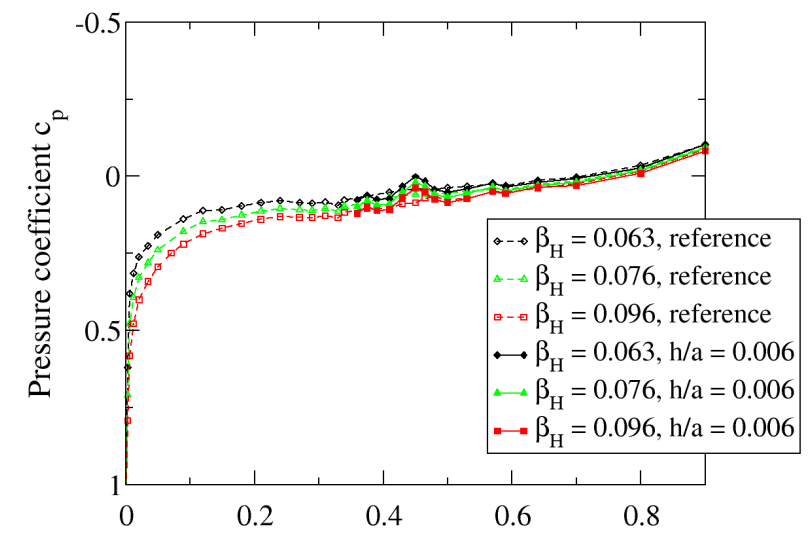

a

Chordwise coordinate $\mathrm{x} / \mathrm{c}$

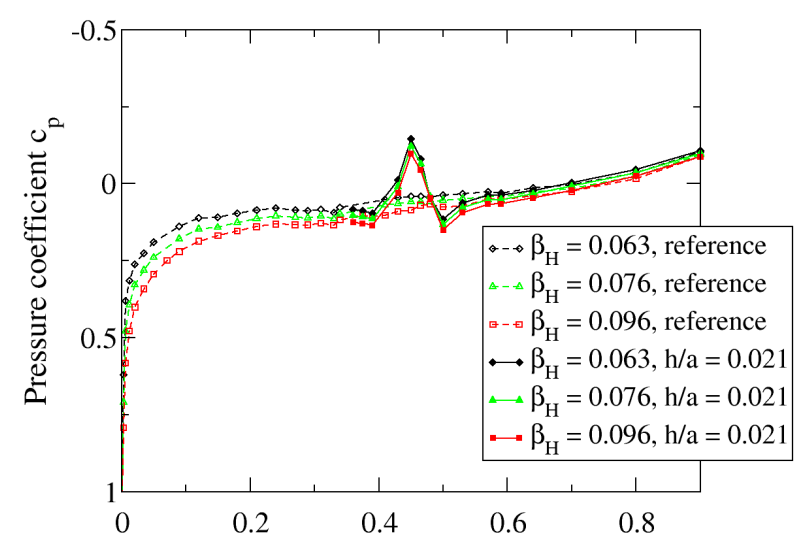

b

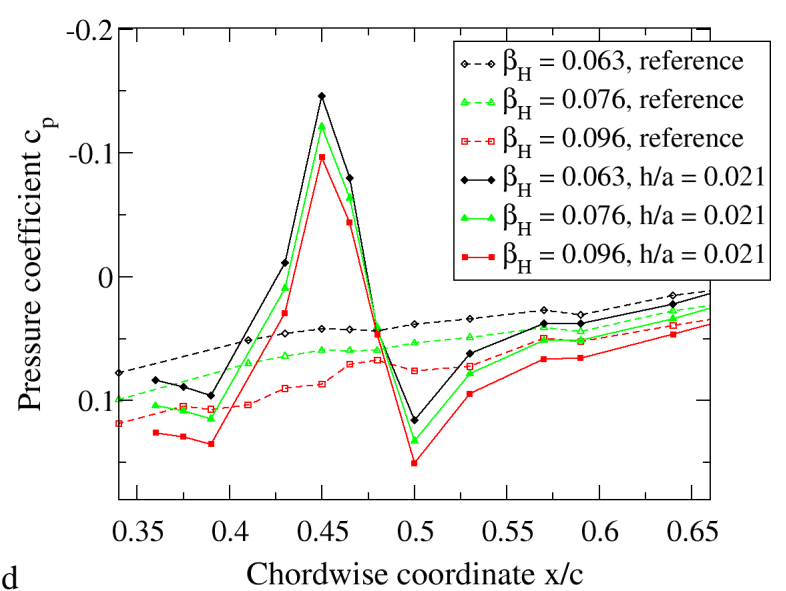

c Chordwise coordinate $\mathrm{x} / \mathrm{c}$

$\mathrm{d}$

Figure 12: Surface pressure distributions measured for the case of Figure 11. a-b: over the whole chord length; c$\mathrm{d}$ : zoomed-in around the bump regions. a, c: cases with the small bump; b, d: cases with the big bump. Start and end of the bumps are located at $x / c=40 \%$ and $50 \%$, respectively.

In the case of the mid bump, the effect of a variation in the global flow acceleration on bump-induced transition was observed to be dependent on Mach and Reynolds numbers. At $\mathrm{M}=0.77$ and $\operatorname{Re} \geq 6 \cdot 10^{6}$, bump-induced transition was essentially unaffected by a change in the global pressure gradient, in a manner similar to that shown above for the big bump. However, at smaller Mach numbers $(\mathrm{M}=0.35$ and 
0.50) and smaller Reynolds numbers $\left(\operatorname{Re}<6 \cdot 10^{6}\right)$, stronger flow acceleration led transition in the presence of the mid bump to occur at a more downstream location - although the downstream shift of the transition location for a certain increase in the Hartree parameter was less pronounced than that observed in the case of the small bump (and, clearly, also of the clean configuration). This is exemplarily shown in Figure 13 by a series of TSP results obtained at $\mathrm{M}=0.50$ and $\mathrm{Re}=4.5 \cdot 10^{6}$. As the Hartree parameter was increased from $\beta_{\mathrm{H}}=0.015$ (Figure 13a) to $\beta_{\mathrm{H}}=0.117$ (Figure 13d), transition was found to move into a more downstream location: from $x_{\mathrm{T}} / c=51.4 \pm 0.1 \%$ to $x_{\mathrm{T}} / c=58.9 \pm 0.7 \%$. For comparison, the boundary layer was found to remain laminar over the whole clean configuration already at $\beta_{\mathrm{H}}=0.095$ (Figure 13c), while transition occurred at $x_{\mathrm{T}} / c=62.4 \pm 0.9 \%$ at $\beta_{\mathrm{H}}=0.015$ (Figure 13c). The different behavior of transition induced by the mid bump, observed for variations in the global pressure gradient at different Mach and Reynolds numbers, will be discussed in more detail in Sec. 5.1. The effect of changes in Mach and Reynolds numbers on transition induced by the small and big bumps will be presented in the next sections.
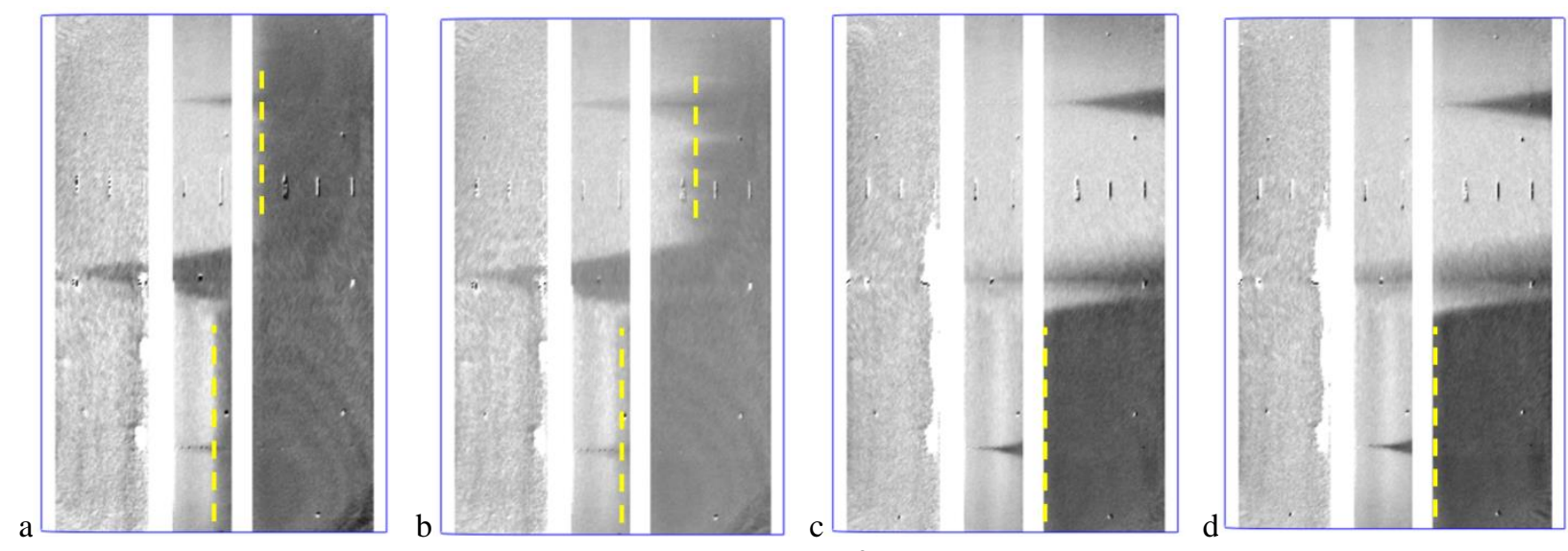

Figure 13: TSP results for different global pressure gradients $\beta_{\mathrm{H}}$ obtained with the clean configuration (bump-free) and with the mid bump $(h / a=0.013)$ at $\mathrm{M}=0.50$ and $\operatorname{Re}=4.5 \cdot 10^{6}$. a: $\beta_{\mathrm{H}}=0.015\left(x_{\mathrm{T}} / c=62.4 \pm 0.9 \%\right.$ vs. $\left.x_{\mathrm{T}} / c=51.4 \pm 0.1 \%\right) ; \mathrm{b}: \beta_{\mathrm{H}}=0.060\left(x_{\mathrm{T}} / c=73.6 \pm 2.0 \%\right.$ vs. $\left.x_{\mathrm{T}} / c=53.5 \pm 0.7 \%\right) ; \mathrm{c}: \beta_{\mathrm{H}}=0.095$ (fully laminar vs. $x_{\mathrm{T}} / c=58.4 \pm 0.2 \%$ ); d: $\beta_{\mathrm{H}}=0.117$ (fully laminar vs. $x_{\mathrm{T}} / c=58.9 \pm 0.7 \%$ ).

\subsection{Effect of the Mach Number}

The influence of a variation in the Mach number on bump-induced transition is shown by the TSP results in Figure 14, which were obtained at a Reynolds number of $\operatorname{Re}=6 \cdot 10^{6}$ and at Hartree parameters in the range $0.060 \leq \beta_{\mathrm{H}} \leq 0.063$. (The Hartree parameter decreased slightly at larger Mach numbers, but the maximal difference between the values of $\beta_{\mathrm{H}}$ obtained at different Mach numbers was less than $5 \%$.) The corresponding surface pressure distributions are shown in Figure 15, where the plots were prepared in a manner analogous to that of the plots presented in Figure 12, but different colors show now the data obtained at different Mach numbers. In the case of the big bump, a change in the Mach number did not lead to significant variations in the transition location (see Figure 14). This finding is in line with the insensitivity of bump-induced transition to changes in the global pressure gradient, which was presented in Sec. 4.2. Also the observed insensitivity of bump-induced transition to variations in the Mach number was very likely due to the marked adverse pressure gradient on the downstream side of the big bump. As shown in Figure 15d, and in agreement with the findings of Masad and Nayfeh (1992) and Masad and Iyer (1993), the local bump-induced variations of the surface pressure distribution were strengthened at larger Mach numbers. As discussed above with regard to the Hartree parameter, the pressure distributions measured on the remaining model surface at different Mach numbers were essentially in agreement - see Figure 15a-b. 
Revision 1. Submitted to the Special Issue of Experimental Thermal and Fluid Science (ETFS) dedicated to the $5^{\text {th }}$ International Conference on Experimental Fluid Mechanics (ICEFM)
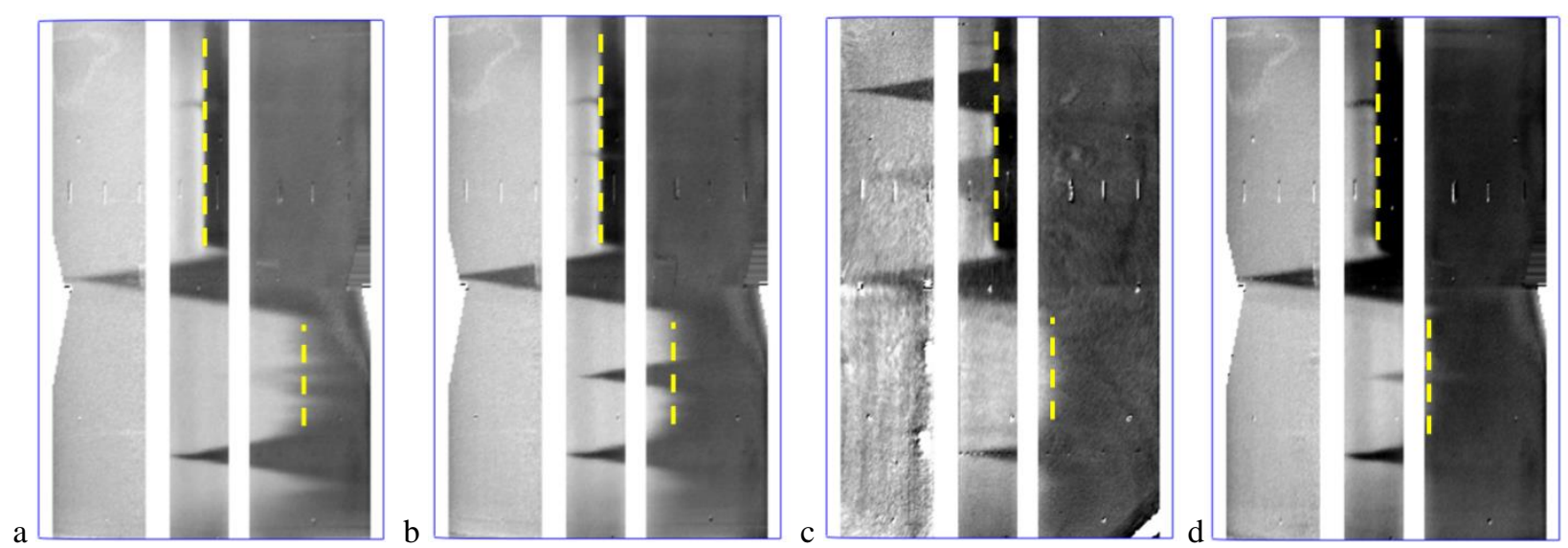

Figure 14: TSP results for different Mach number obtained with the small $(h / a=0.006)$ and big $(h / a=0.021)$ bumps at $\operatorname{Re}=6 \cdot 10^{6}$ and $0.060 \leq \beta_{\mathrm{H}} \leq 0.063 . \mathrm{a}: \mathrm{M}=0.35\left(x_{\mathrm{T}} / c=76.1 \pm 1.1 \%\right.$ vs. $\left.x_{\mathrm{T}} / c=48.8 \pm 0.2 \%\right) ; \mathrm{b}: \mathrm{M}=0.50$ $\left(x_{\mathrm{T}} / c=67.8 \pm 0.6 \%\right.$ vs. $\left.x_{\mathrm{T}} / c=48.7 \pm 0.2 \%\right) ; \mathrm{c}: \mathrm{M}=0.65\left(x_{\mathrm{T}} / c=64.2 \pm 0.4 \%\right.$ vs. $\left.x_{\mathrm{T}} / c=48.5 \pm 0.5 \%\right) ; \mathrm{d}: \mathrm{M}=0.77$ $\left(x_{\mathrm{T}} / c=61.9 \pm 0.1 \%\right.$ vs. $\left.x_{\mathrm{T}} / c=47.7 \pm 0.5 \%\right)$.
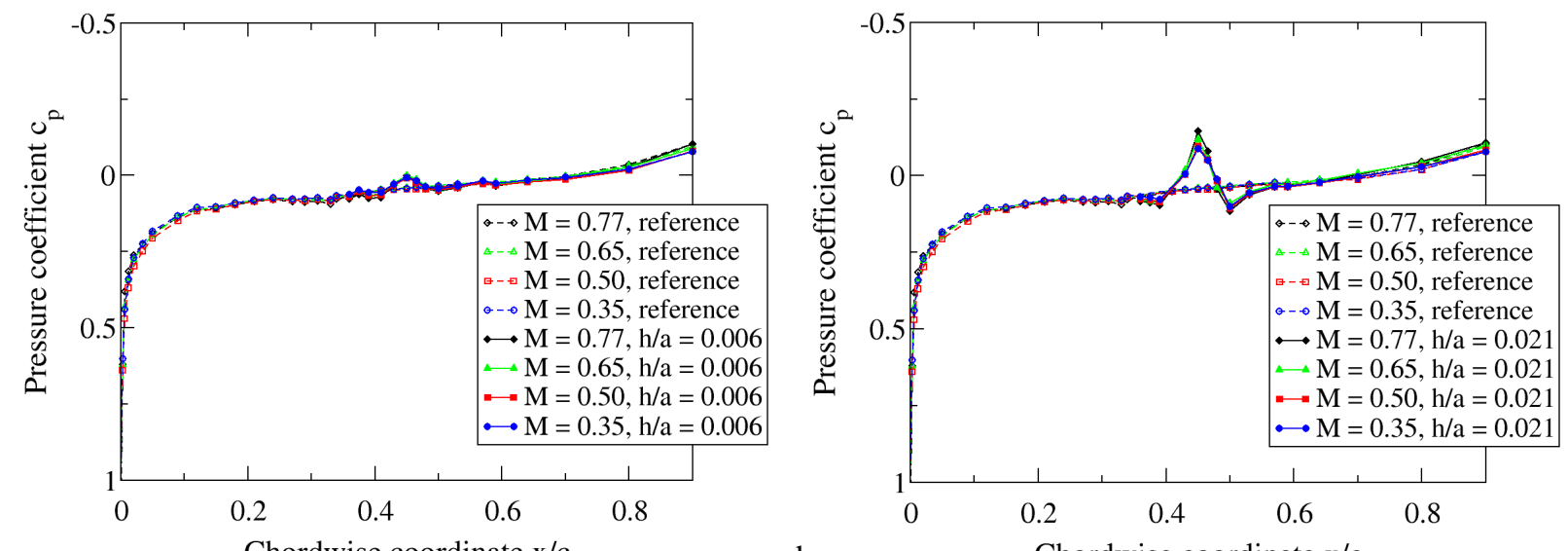

a

Chordwise coordinate $\mathrm{x} / \mathrm{c}$

$\mathrm{b}$

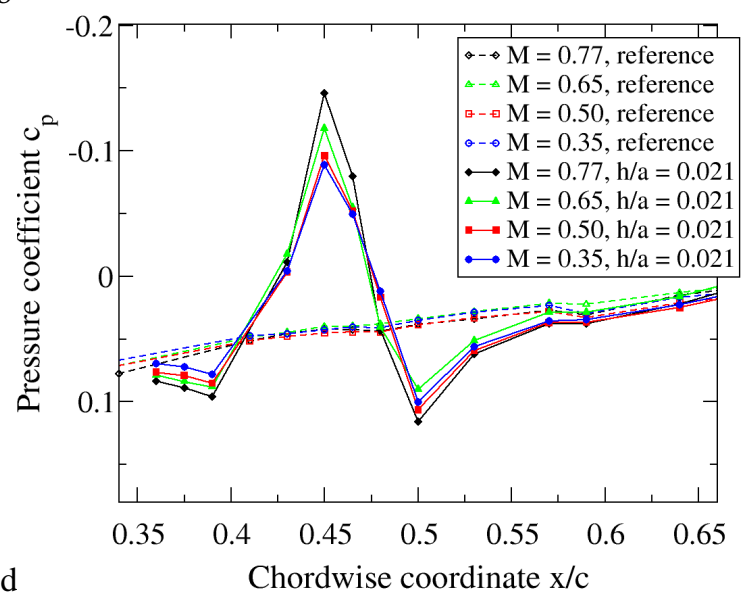

c

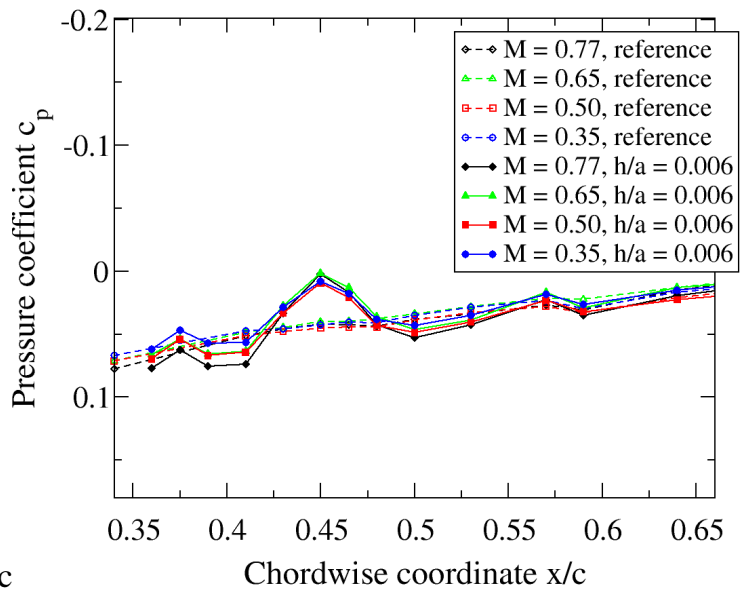

d

Figure 15: Surface pressure distributions measured for the case of Figure 14. a-b: over the whole chord length; cd: zoomed-in around the bump regions. a, c: cases with the small bump; b, d: cases with the big bump. Start and end of the bumps are located at $x / c=40 \%$ and $50 \%$, respectively.

The more pronounced adverse pressure gradient on the downstream side of the big bump led to the small upstream shift of the transition location at larger Mach numbers $\left(\Delta\left(x_{\mathrm{T}} / c\right) \sim 1 \%\right)$. In contrast, in the case of the small bump, an increase in the Mach number led to a larger upstream shift of the transition location: 
Revision 1. Submitted to the Special Issue of Experimental Thermal and Fluid Science (ETFS) dedicated to the $5^{\text {th }}$ International Conference on Experimental Fluid Mechanics (ICEFM)

from $x_{\mathrm{T}} / c=76.1 \pm 1.1 \%$ at $\mathrm{M}=0.35$ (Figure $14 \mathrm{a}$ ) to $x_{\mathrm{T}} / c=61.9 \pm 0.1 \%$ at $\mathrm{M}=0.77$ (Figure $14 \mathrm{~d}$ ). Also the local variations in the pressure distribution induced by the small bump were strengthened by an increase in the Mach number (see Figure 15c), but this effect was clearly less pronounced than that observed in the presence of the big bump. Although the more marked adverse pressure gradient on the downstream side of the small bump probably contributed to the upstream movement of the transition location, the most important variation leading to this result is expected to be the increased level of external disturbances in DNW-KRG at larger Mach numbers (see Koch (2004), Costantini (2016), Risius et al. (2018) and Risius (2018)): earlier transition was likely induced by the related increase (via the receptivity process) in the initial amplitude of the boundary-layer disturbances. The increased wall temperature ratio $T_{w} / T_{a w}$ at larger Mach numbers (see Sec. 3) was also likely to contribute appreciably to the observed upstream shift of the transition location.

\subsection{Effect of the Chord Reynolds Number}

The effect of the chord Reynolds number (i.e., of the unit Reynolds number) is presented here for test conditions at $\mathrm{M}=0.77$ and $\beta_{\mathrm{H}}=0.096$. The TSP results and the corresponding pressure distributions are shown in Figure 16 and Figure 17, respectively. The plots of the surface pressure distributions are presented in a manner analogous to that of the plots in Figure 12 and Figure 15; in Figure 17, however, different colors show the data obtained at different Reynolds numbers. Transition induced by the small bump was found to move into a more upstream location as the chord Reynolds number was increased: from $x_{\mathrm{T}} / c=82.6 \pm 2.0 \%$ at $\mathrm{Re}=6 \cdot 10^{6}$ (Figure 16a) to $x_{\mathrm{T}} / c=58.8 \pm 0.2 \%$ at $\operatorname{Re}=10 \cdot 10^{6}$ (Figure 16c). This effect of a larger chord Reynolds number on boundary-layer transition in the presence of surface bumps has been observed since the earliest work of Fage (1943) and Carmichael (1959). Note also that the number of turbulent wedges in the TSP results increased significantly at larger Reynolds numbers. Besides the mid-span area, where one and two additional turbulent wedges were observed to originate from the leading-edge region as the chord Reynolds number was increased to $\operatorname{Re}=8$ and $10 \cdot 10^{6}$, respectively, all other additional turbulent wedges were seen to arise from the upstream junctions between model parts (located at $x / c=35$ and $37 \%$, see Figure 2). These turbulent wedges were probably due to disturbances generated by micron-sized, three-dimensional roughness elements (see Sec. 2.2), which relative size (with respect to the boundary-layer thickness) increases at larger Reynolds numbers; they therefore generate stronger disturbances and are more likely to trigger premature transition (see, e.g., Braslow (1966)). This was the very probable reason for the observed increase of the number of turbulent wedges in the TSP results. In particular, the turbulent wedges superimposed onto the bump-induced transition front made impossible to detect "natural" transition in the presence of the big bump at $\operatorname{Re}=10 \cdot 10^{6}$ (Figure 16c). The detected transition location was at $x_{\mathrm{T}} / c=42.4 \pm 0.8 \%$, i.e., upstream of the bump crest. For this case, the "natural" transition location is also expected to be close to that measured in the presence of the big bump at the two lower Reynolds numbers. The upstream shift in the transition location observed at $h / a=0.021$ for an increase in the chord Reynolds number from $\mathrm{Re}=6$ to $8 \cdot 10^{6}$ was approximately $\Delta\left(x_{\mathrm{T}} / c\right)=1 \%$ (see Figure 16a-b). As discussed in the previous sections, on the downstream side of the big bump the boundary layer was found to separate; transition in the laminar separation bubble was only weakly influenced by a change in the Reynolds number, leading to this small variation in the transition location. According to Masad and Iyer (1993) and Masad and Malik (1995), the boundary layer is expected to separate at a slightly more upstream location at larger Reynolds numbers. This effect may have contributed to the observed (small) upstream shift of the transition location. It should be remarked here that, at larger chord Reynolds numbers, the Reynolds number based on the chordwise location of the bump crest $\operatorname{Re}_{x \mathrm{~b}}=x_{\mathrm{b}} U_{\infty} / v_{\infty}$ (with $x_{\mathrm{b}} / c=45 \%$ ) was also larger. This aspect will be further discussed in Sec. 5.3.

As can be seen in Figure 17, the surface pressure distributions were essentially unaffected by changes in the Reynolds number. The small variations observed around $x / c=40 \%$ in the case of the small bump (Figure 17c) and on the downstream side of the big bump (Figure 17d) may be related to a slightly different effect of the bumps on the boundary-layer development (because of the different bump size 
Revision 1. Submitted to the Special Issue of Experimental Thermal and Fluid Science (ETFS) dedicated to the $5^{\text {th }}$ International Conference on Experimental Fluid Mechanics (ICEFM)

relative to, e.g., the boundary-layer displacement thickness), but may be also due to the influence of the additional turbulent wedges arising in the vicinity of the pressure tap rows.
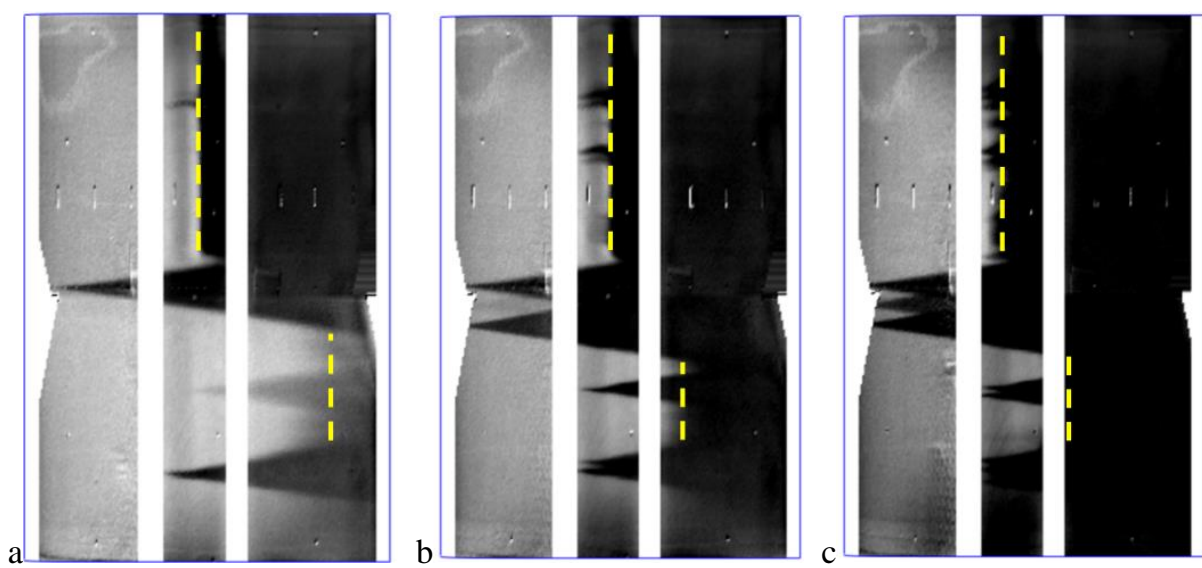

Figure 16: TSP results for different chord Reynolds numbers obtained with the small $(h / a=0.006)$ and big ( $h / a=0.021)$ bumps at $\mathrm{M}=0.77$ and $\beta_{\mathrm{H}}=0.096$. a: $\mathrm{Re}=6 \cdot 10^{6}\left(x_{\mathrm{T}} / c=82.6 \pm 2.0 \%\right.$ vs. $\left.x_{\mathrm{T}} / c=48.7 \pm 0.2 \%\right)$;

b: $\operatorname{Re}=8 \cdot 10^{6}\left(x_{\mathrm{T}} / c=64.2 \pm 0.3 \%\right.$ vs. $\left.x_{\mathrm{T}} / c=47.8 \pm 0.4 \%\right) ; \mathrm{c}: \operatorname{Re}=10 \cdot 10^{6}\left(x_{\mathrm{T}} / c=58.8 \pm 0.2 \%\right.$ vs. $\left.x_{\mathrm{T}} / c=42.4 \pm 0.8 \%\right)$.
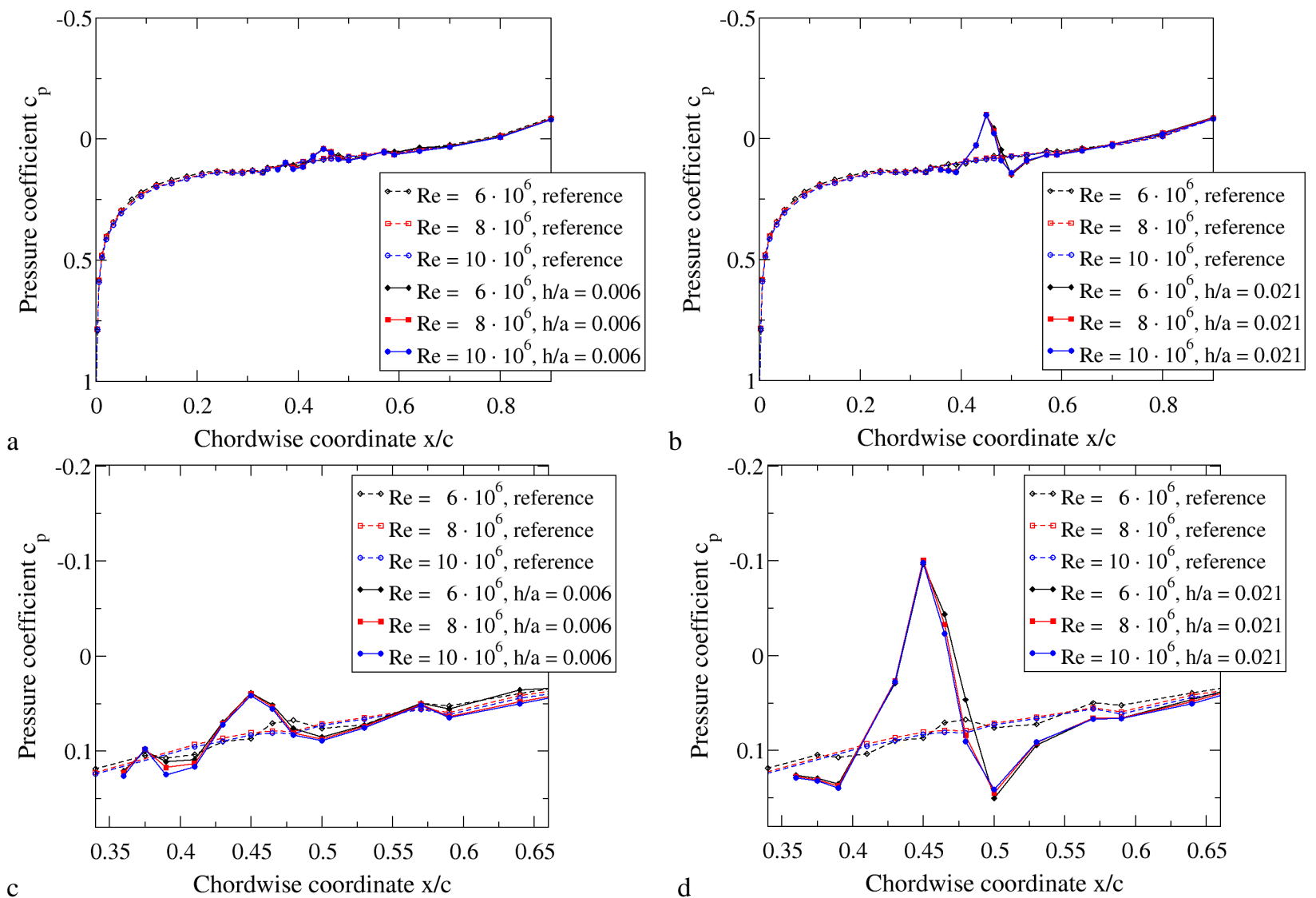

Figure 17: Surface pressure distributions measured for the case of Figure 16. a-b: over the whole chord length; cd: zoomed-in around the bump regions. a, c: cases with the small bump; b, d: cases with the big bump. Start and end of the bumps are located at $x / c=40 \%$ and $50 \%$, respectively. 
Revision 1. Submitted to the Special Issue of Experimental Thermal and Fluid Science (ETFS) dedicated to the $5^{\text {th }}$ International Conference on Experimental Fluid Mechanics (ICEFM)

\section{Discussion}

The experimental results will be discussed in different sections, where the influence of the bump heightto-length ratio on boundary-layer transition will be analyzed in combination with the influence of changes in another parameter. The other two parameters will be maintained fixed in the discussion, in order to facilitate the presentation of the effects on transition of the two varied parameters.

The transition Reynolds number $\operatorname{Re}_{x \mathrm{~T}}=x_{\mathrm{T}} U_{\infty} / v_{\infty}$ will be used to quantify the influence of the parameters on boundary-layer transition. Note that in several cases, such as those shown in Figure 6a and Figure 13c$\mathrm{d}$, the boundary layer remained laminar over the whole clean (i.e., bump-free) surface. In these cases, the reference transition Reynolds numbers $\mathrm{Re}_{x \mathrm{~T}, 0}$ for a nominally smooth surface were taken from the results of previous studies (Costantini et al. (2016), Costantini (2016) and Costantini et al. (2018)), in which the same model contour had been examined at larger chord Reynolds numbers. This was possible because the values of $\mathrm{Re}_{x \mathrm{~T}, 0}$ obtained in different investigations were verified to be in agreement for the test conditions at which transition occurred over the model surface (see Sec. 4). In those previous studies, only one junction was present on the model upper surface, instead of the three junctions (two upstream of the bump) on the configuration investigated in this work, and the number of turbulent wedges arising at that only one junction at larger Reynolds numbers was significantly smaller than that observed here. Therefore, chord Reynolds numbers larger than those examined in the present work could be considered in Costantini et al. (2016), Costantini (2016) and Costantini et al. (2018); at those Reynolds numbers, transition was shown to occur over the model upper surface even at the largest examined Hartree parameters. Another finding of those previous investigations was that, for the examined clean configuration, the transition Reynolds number was essentially independent of the chord Reynolds number. This observation is reasonably assumed to hold also for the present clean configuration, thus enabling the use of the $\mathrm{Re}_{x \mathrm{~T}, 0}$-values from earlier work as reference transition Reynolds numbers in the present work.

\subsection{Combined Effect of Bump Height-to-Length Ratio and Global Pressure Gradient}

The effect of the bump height-to-length ratio $h / a$ on boundary-layer transition is analyzed in this section in combination with the influence of the Hartree parameter $\beta_{\mathrm{H}}$. The considered test cases are those presented in Sec. 4.2. The results obtained at the examined test conditions, including also those obtained with the mid-bump configuration, are summarized in Figure 18. In this figure, the transition Reynolds number is plotted as a function of the bump height-to-length ratio, and data obtained at different global pressure gradients are shown by symbols with different colors. This representation of the results enables a direct comparison with correlations and criteria for critical bump size from previous work, where the "critical" bump height-to-length ratios were defined as "those which produce transition forward [i.e., upstream] of the location where it would occur in the absence of the surface imperfection" (see Holmes et al. (1985)). In any case, the ratios between the bump height $h$ and the local boundary-layer displacement thickness $\delta_{1, \mathrm{~b}}$ for the corresponding data points of Figure 18 are reported in Table $3 . \delta_{1, \mathrm{~b}}$ is here the value of the displacement thickness at the bump crest location $x_{\mathrm{b}} / c$ but for the clean (bump-free) configuration; it was determined via boundary-layer computations carried out in the same manner as that described in Costantini et al. (2016) and Costantini (2016). In Figure 18, the critical $h / a$ provided as a function of $\operatorname{Re}_{x \mathrm{~T}}$ by the correlation of Fage (1943) is shown by a dashed-dotted brown line; the critical bump-to-height ratio derived from the criterion provided by Carmichael (1959) is indicated by a magenta dashed line; the transition Reynolds numbers predicted in Masad and Iyer (1993) using the $\mathrm{e}^{\mathrm{N}_{\mathrm{T}}}$ method (with $\mathrm{N}_{\mathrm{T}}=9$ ) are shown by the violet symbols; and the transition Reynolds numbers determined experimentally in Perraud et al. (2004) are shown by orange symbols. (The reported values of $\operatorname{Re}_{x \mathrm{~T}}$ were obtained in Masad and Iyer (1993) at $M=0.8$, i.e., at a Mach number slightly larger than $M=0.77$ of the present data.) Moreover, the Reynolds number based on the chordwise location of the bump crest $\operatorname{Re}_{x \mathrm{~b}}=x_{\mathrm{b}} U_{\infty} / v_{\infty}$ is indicated in Figure 18 by a dotted grey line. Error bars are shown for all current data points, but they are in most cases smaller than the symbols and therefore not easily visible. 
Revision 1. Submitted to the Special Issue of Experimental Thermal and Fluid Science (ETFS) dedicated to the $5^{\text {th }}$ International Conference on Experimental Fluid Mechanics (ICEFM)

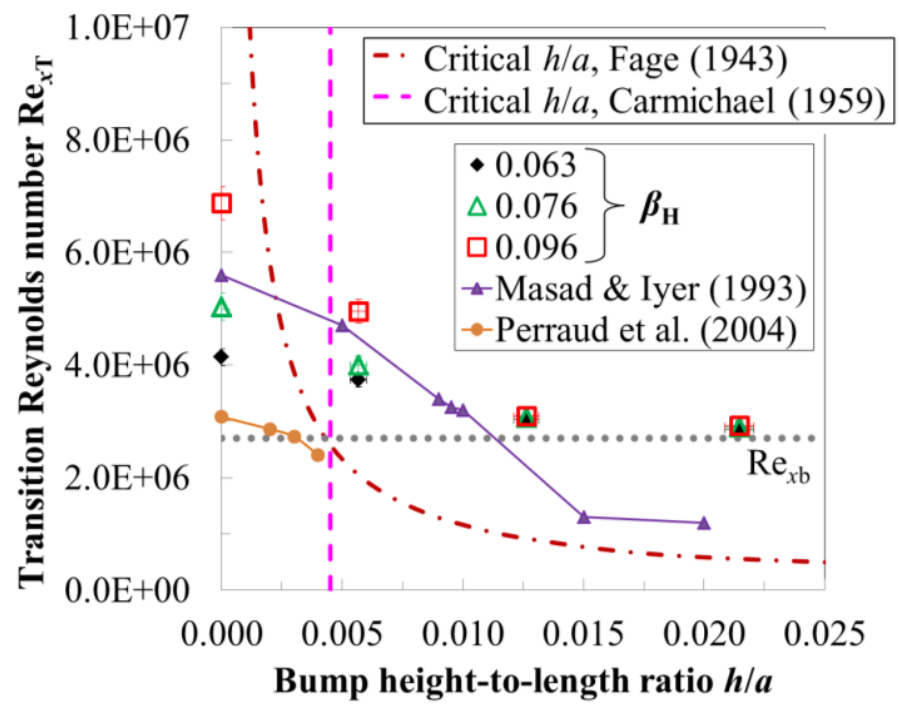

Figure 18: Transition Reynolds number as a function of the bump height-to-length ratio for $\mathrm{M}=0.77$ and $\operatorname{Re}=6 \cdot 10^{6}$.

Table 3: Bump height relative to local boundary-layer displacement thickness for the data points of Figure 18.

\begin{tabular}{|c|c|c|c|}
\hline $\boldsymbol{\beta}_{\mathbf{H}}$ & $\begin{array}{c}\boldsymbol{h} / \boldsymbol{\delta}_{\mathbf{1}, \mathbf{b}} \\
(\boldsymbol{h} / \boldsymbol{a}=\mathbf{0 . 0 0 6})\end{array}$ & $\begin{array}{c}\boldsymbol{h} / \boldsymbol{\delta}_{\mathbf{1}, \mathbf{b}} \\
(\boldsymbol{h} / \boldsymbol{a}=\mathbf{0 . 0 1 3})\end{array}$ & $\begin{array}{c}\boldsymbol{h} / \boldsymbol{\delta}_{\mathbf{1}, \mathbf{b}} \\
(\boldsymbol{h} / \boldsymbol{a}=\mathbf{0 . 0 2 1})\end{array}$ \\
\hline 0.063 & 1.11 & 2.48 & 4.21 \\
\hline 0.076 & 1.13 & 2.51 & 4.26 \\
\hline 0.096 & 1.15 & 2.56 & 4.34 \\
\hline
\end{tabular}

A glance at Figure 18 clearly shows that the transition Reynolds number already decreased for the configuration with the bump with height-to-length ratio $h / a=0.006$, as compared to its value measured for the clean configuration. In a manner analogous to that described in Nayfeh et al. (1988), Cebeci and Egan (1989), Masad and Nayfeh (1992), Arnal (1992), Masad and Iyer (1993) and Perraud et al. (2004), this result was most likely due to the enhanced amplification of the boundary-layer disturbances in the local adverse pressure gradient regions upstream of the bump and, more importantly, on the downstream side of the bump (see Secs. 4.1 and 4.2). This adverse effect on boundary-layer stability appeared to be more pronounced than the favorable effect of the enhanced flow acceleration over the upstream side of the bump and in the region downstream of the bump (recovery region), thus inducing earlier transition, as compared to the clean configuration. As shown in Figure 6, an increase of the bump height-to-length ratio to $h / a=0.013$ led to a strengthening of the local pressure gradients induced by the bump; the related enhancement of the bump-induced amplification of the boundary-layer disturbances very likely led to a further decrease of the transition Reynolds number. At the considered Mach number $M=0.77$, transition was found for this bump configuration approximately at the downstream end of the bump for all three examined global pressure gradients. This is shown in Figure 18 by the vicinity of the corresponding symbols (those at $h / a=0.013$ ) to the dotted gray line. At the transition location, the global pressure gradient had not been recovered yet; in fact, the transition process started in the region of adverse pressure gradient over the downstream side of the bump. At $h / a=0.021$, transition occurred even closer to the bump crest, well inside the region of adverse pressure gradient; the upstream shift of the transition location (and therefore the reduction of $\mathrm{Re}_{x \mathrm{~T}}$ ) was small, with respect to its value at $h / a=0.013$. As discussed in Nayfeh et al. (1988), Cebeci and Egan (1989), Arnal (1992) and Perraud et al. (2004), the streamwise distributions of the amplification factors ( $\mathrm{N}$-factors) of Tollmien-Schlichting waves are steep in the examined region. This is illustrated in Figure 19, where the dotted grey line qualitatively shows the streamwise distribution of the $\mathrm{N}$-factors over a smooth surface, while the expected effect of the small, mid and big bumps on the $\mathrm{N}$-factor evolution is shown by the green, red and blue lines, respectively. Assuming 
Revision 1. Submitted to the Special Issue of Experimental Thermal and Fluid Science (ETFS) dedicated to the $5^{\text {th }}$ International Conference on Experimental Fluid Mechanics (ICEFM)

that transition occurs at the same $\mathrm{N}$-factor for all configurations, the enhancement of the disturbance amplification due to the mid bump is expected to lead to a significant upstream movement of the transition location, whereas an increase of the bump height-to-length ratio from $h / a=0.013$ to 0.021 is expected to induce only a small upstream shift of the transition location. These expectations are in line with the present experimental observations.

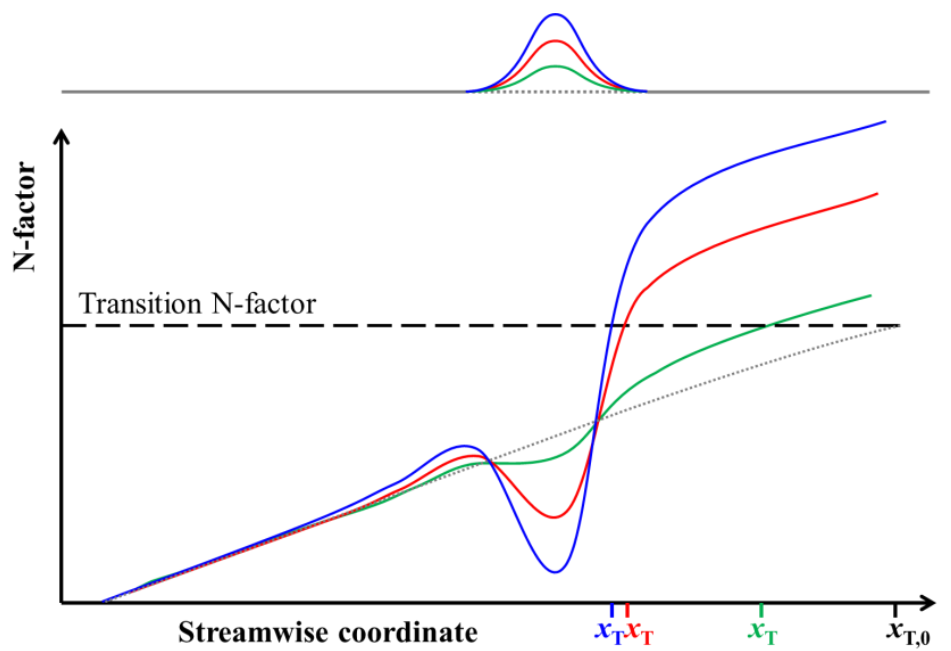

Figure 19: Schematic illustrating the effect of bumps of different height on the amplification factors of TollmienSchlichting waves. The expected upstream shift of the transition location (as it would be predicted according to the $\mathrm{e}^{\mathrm{N}_{\mathrm{T}}}$ method) is also shown.

The effect of the global pressure gradient on boundary-layer transition can be also seen in Figure 18. The favorable effect of larger Hartree parameters on transition over a smooth surface is well known (see, e.g., Arnal (1992), Schlichting and Gersten (2000), Costantini et al. 2016, Costantini (2016), Risius et al. (2018) and Risius (2018)), but larger transition Reynolds numbers were achieved at larger $\beta_{\mathrm{H}}$ also with the small-bump configuration. The relative increase in $\mathrm{Re}_{x \mathrm{~T}}$ for a certain increase in Hartree parameter was, however, smaller than that found for the clean configuration. The attenuation of the favorable influence of the streamwise pressure gradient on boundary-layer transition was very likely due to the bump-induced amplification of the boundary-layer disturbances, in a manner analogous to that discussed in Costantini (2016) for a step-induced amplification. As shown in Figure 12, the bump-induced pressure gradients were approximately of the same magnitude for all considered global pressure gradients; the corresponding bump-induced variations of the disturbance amplification factors can be also assumed to be nearly independent of the global pressure gradient (at least to a first approximation). In the presence of the bump with $h / a=0.006$, an increase in the Hartree parameter is still expected to reduce the disturbance amplification in the regions at sufficient distance from the bump, but its relative effect on the overall amplification of the disturbances is expected to become weaker, since the bump-induced amplification has remained approximately unchanged. This provides an explanation for the reduced sensitivity of boundarylayer transition to variations in the Hartree parameter observed in the presence of the small bump. With the mid and big bumps, the transition Reynolds number was essentially insensitive to variations in the global pressure gradient. As discussed above, transition occurred in these cases in the region of adverse pressure gradient over the downstream side of the bump, where the gradient of the $\mathrm{N}$-factor curves is very marked (see schematic in Figure 19). The effect of the global pressure gradient on the steep N-factor curves in the examined region is presumably small, but even if this effect would be larger, the corresponding variation in transition location is expected to be small (provided that the transition $\mathrm{N}$-factor has remained unchanged). This is especially the case when transition occurs within a laminar separation bubble, as it was demonstrated for the big-bump configurations. These expectations from considerations based on linear stability theory are in line with the experimental observations reported in the present work. 
Revision 1. Submitted to the Special Issue of Experimental Thermal and Fluid Science (ETFS) dedicated to the $5^{\text {th }}$ International Conference on Experimental Fluid Mechanics (ICEFM)

The other test case presented in Sec. 4.2 was with the clean / mid-bump configuration at $\mathrm{M}=0.50$ and $\operatorname{Re}=4.5 \cdot 10^{6}$ (see Figure 13). The results obtained at the examined test conditions, including also those obtained with the small- / big-bump configuration, are summarized in Figure 20, in a manner similar to that of Figure 18 for the previous test case. The values of the bump height $h$ relative to the local boundarylayer displacement thickness $\delta_{1, \mathrm{~b}}$ are reported in Table 4 for the corresponding data points of Figure 20. In the presence of the small bump, the boundary layer remained laminar over the whole model upper surface at the largest Hartree parameters $\left(\beta_{\mathrm{H}}=0.095\right.$ and 0.117$)$. The value of $x_{\mathrm{T}} / c=100 \%$ was taken to evaluate the transition Reynolds number, but this could be even larger. The corresponding data points in Figure 20 are enclosed by black circles. In general, the trends observed at these test conditions were analogous to those discussed above with regard to the cases at $\mathrm{M}=0.77$ and $\operatorname{Re}=6 \cdot 10^{6}$ : the only difference in the behavior of bump-induced transition for variations in the global pressure gradient was observed in the presence of the mid bump. As shown in Figure 13, an increase in the Hartree parameter from $\beta_{\mathrm{H}}=0.015$ to 0.117 did lead to a downstream shift of the transition location transition at $\mathrm{M}=0.50$ and $\operatorname{Re}=4.5 \cdot 10^{6}$. This result can be seen also in terms of the increase in the transition Reynolds number in Figure 20 (symbols at $h / a=0.013$ ). At this lower Mach number, the bump-induced variation of the pressure distribution was less pronounced than that at $M=0.77$; this can be seen in Figure 15 for the small and big bump configurations. Since the adverse pressure gradient over the downstream side of the bump was weaker at $\mathrm{M}=0.50$, and the chord Reynolds number was lower $\left(\operatorname{Re}=4.5 \cdot 10^{6}\right.$, instead of $6 \cdot 10^{6}$ at $\mathrm{M}=0.77$ ), transition occurred - even at $\beta_{\mathrm{H}}=0.015$ - in the recovery region downstream of the mid bump, where a variation in the global pressure gradient had maintained an appreciable influence on boundarylayer transition. (As qualitatively shown in in Figure 19, the gradients of the $\mathrm{N}$-factor curves in the recovery region are reduced, as compared to those in the local adverse pressure gradient region.) In fact, an increase in the Hartree parameter led in this case to an increase in the transition Reynolds number; for a certain increase in $\beta_{\mathrm{H}}$, however, the increase in $\operatorname{Re}_{x \mathrm{~T}}$ was smaller than that for the configurations with $h / a=0$ and 0.006 . It should be also reminded here that a difference in the boundary-layer evolution in terms of separation on the downstream side of the mid bump cannot be excluded. As discussed in Sec. 4.1, it is possible that, at $h / a=0.013$, the boundary layer separated for $\mathrm{M}=0.77$, while it may have been remained attached at $M=0.50$. Clearly, this difference in the boundary-layer evolution would have further enhanced the aforementioned difference in the transition behavior in the presence of the mid bump.

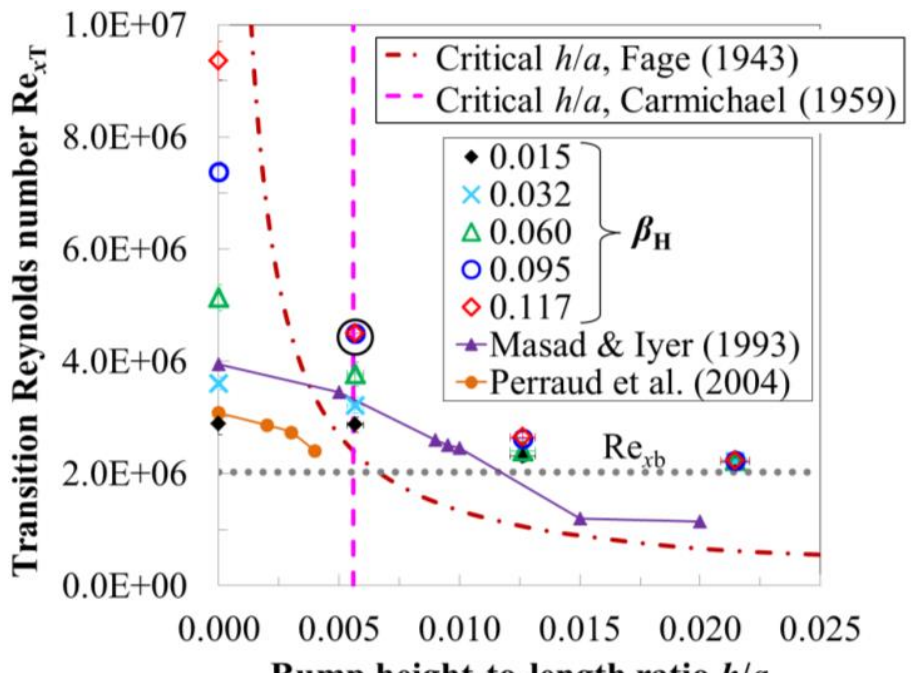

Figure 20: Transition Reynolds number as a function of the bump height-to-length ratio for $\mathrm{M}=0.50$ and $\operatorname{Re}=4.5 \cdot 10^{6}$. 
Revision 1. Submitted to the Special Issue of Experimental Thermal and Fluid Science (ETFS) dedicated to the $5^{\text {th }}$ International Conference on Experimental Fluid Mechanics (ICEFM)

Table 4: Bump height relative to local boundary-layer displacement thickness for the data points of Figure 20.

\begin{tabular}{|c|c|c|c|}
\hline $\boldsymbol{\beta}_{\mathbf{H}}$ & $\begin{array}{c}\boldsymbol{h} / \boldsymbol{\delta}_{\mathbf{1 , b}} \\
(\boldsymbol{h} / \boldsymbol{a}=\mathbf{0 . 0 0 6})\end{array}$ & $\begin{array}{c}\boldsymbol{h} / \boldsymbol{\delta}_{\mathbf{1 , b}} \\
(\boldsymbol{h} / \boldsymbol{a}=\mathbf{0 . 0 1 3})\end{array}$ & $\begin{array}{c}\boldsymbol{h} / \boldsymbol{\delta}_{\mathbf{1 , b}} \\
(\boldsymbol{h} / \boldsymbol{a}=\mathbf{0 . 0 2 1})\end{array}$ \\
\hline 0.015 & 0.97 & 2.17 & 3.68 \\
\hline 0.032 & 0.99 & 2.21 & 3.75 \\
\hline 0.060 & 1.04 & 2.33 & 3.95 \\
\hline 0.095 & 1.06 & 2.37 & 4.02 \\
\hline 0.117 & 1.07 & 2.39 & 4.05 \\
\hline
\end{tabular}

A look at Figure 18 and Figure 20 shows that the observed decrease in the transition Reynolds number for a certain increase in the bump height-to-length ratio was qualitatively similar to that predicted in Masad and Iyer (1993). The critical imperfection values provided by the criterion of Carmichael (1959) are close to the height-to-length ratio of the small bump $(h / a=0.006)$, especially in the case at $\mathbf{M}=0.50$, while the critical values of $h / a$ provided by the correlation of Fage (1943) are smaller. For most of the global pressure gradients examined in the present experiments, a significant reduction of the transition Reynolds number was found at $h / a=0.006$, as compared to the value of $\operatorname{Re}_{x \mathrm{~T}, 0}$ for the clean configuration. This result is in qualitative agreement with the predictions based on the criterion of Fage (1943), while the critical value of $h / a$ seems to be generally overpredicted by the criterion of Carmichael (1959). In Perraud et al. (2004), a significant reduction of the transition Reynolds number was already observed for $h / a \leq 0.004$. At this point of the discussion, it should be reminded that the bump height-to-length ratio alone is not sufficient to completely describe the effect of bumps on boundary-layer transition: there are a number of other factors that have an influence on the value of the transition Reynolds number (global pressure gradient, freestream turbulence, model surface quality, compressibility, location and shape of the imperfection, among others). In Carmichael (1959), the imperfection had the form of a sinusoidal wave, instead of the sinusoidal bump examined in Fage (1943), Perraud et al. (2004) and in the present work; the contour of the bump mainly considered in Masad and Iyer (1993) was a cubic function of the streamwise coordinate. A high subsonic Mach number $(M=0.8)$ was studied in Masad and Iyer (1993), whereas the Mach number was "low to moderate" in Fage (1943) and Carmichael (1959). Slightly and strongly stabilized boundary layers (via flow acceleration and wall suction) were considered in the experiments reported in Fage (1943) and Carmichael (1959), respectively. As discussed in Holmes (1985), the criterion of Carmichael (1959) is therefore regarded to apply for boundary layers under a similar degree of (strong) stabilization, while it is expected to "overpredict the allowable waviness in a region of unaccelerated flow". The criterion of Fage (1943) is recommended in cases with nearly-zero pressure gradient. The prediction of Masad and Iyer (1993) was obtained for a zero global pressure gradient, while the variation of the transition Reynolds number was measured in Perraud et al. (2004) on an NLF supercritical airfoil model at a negative angle-of-attack, i.e., with a favorable global pressure gradient. As can be seen from Figure 18 and Figure 20, a reduction in the transition Reynolds number was observed in both previous investigations at values of $h / a$ smaller than the critical value from Carmichael (1959), while the critical value of $h / a$ from Fage (1943) appears to be in qualitative agreement with the reduction in the transition Reynolds number predicted in Masad and Iyer (1993). In fact, the reduction in $\mathrm{Re}_{x \mathrm{~T}}$ with increasing $h / a$ was more pronounced for the data of Perraud et al. (2004), i.e., for an accelerated flow, than for the data of Masad and Iyer (1993), where the global pressure gradient was zero. Also in the present work, the reduction in the transition Reynolds number with increasing $h / a$ was more marked at larger values of $\beta_{\mathrm{H}}$ (e.g., at $\beta_{\mathrm{H}}=0.096$ and 0.117 for $\mathrm{M}=0.77$ and 0.50 , respectively) than that observed at smaller Hartree parameters (e.g., at $\beta_{\mathrm{H}}=0.063$ and 0.015 for $\mathrm{M}=0.77$ and 0.50 , respectively). The global pressure gradient thus appears to have a fundamental role in the sensitivity of boundary-layer transition to changes in the imperfection height-to-length ratio. The increased transition sensitivity with stronger global flow acceleration can be illustrated by considering the reduced streamwise gradient of the $\mathrm{N}$-factor curves of Tollmien-Schlichting waves in the region at sufficient distance from the bumps, in a manner analogous to that discussed in Costantini (2016) for the increased transition sensitivity in the presence of forward- 
Revision 1. Submitted to the Special Issue of Experimental Thermal and Fluid Science (ETFS) dedicated to the $5^{\text {th }}$ International Conference on Experimental Fluid Mechanics (ICEFM)

facing steps at larger Hartree parameters. Assuming that the bump-induced increase in the $\mathrm{N}$-factors is approximately the same for the examined global pressure gradients, and also that the transition $\mathrm{N}$-factor remains approximately unchanged, the expected upstream shift of the transition location for a certain value of $h / a$ is expected to be larger for a flatter $\mathrm{N}$-factor curve (i.e., at larger $\beta_{\mathrm{H}}$ ) than for a steeper one (i.e., at smaller $\beta_{\mathrm{H}}$ ). This expectation is in line with the trends presented in Figure 18 and Figure 20.

Concluding this section, the critical values of $h / a$ from the criteria of Fage (1943) and Carmichael (1959) appear to be useful first estimations for the effect of bumps and waviness on boundary-layer transition, but the actual global pressure gradient must be considered for a more accurate estimation of the variation of the transition location as a function of the imperfection dimension.

\subsection{Combined Effect of Bump Height-to-Length Ratio and Mach Number}

The combined effect of $h / a$ and Mach number on boundary-layer transition is discussed in this section for the test conditions presented in Sec. 4.3. The results obtained at $M=0.35$ to 0.77 , including also those achieved with the mid-bump configurations, are summarized in Figure 21. This figure is prepared in a manner analogous to Figure 18 and Figure 20, but different symbols show here data obtained at different Mach numbers. The ratios between the bump height $h$ and the local boundary-layer displacement thickness $\delta_{1, \mathrm{~b}}$ for the corresponding data points of Figure 21 are reported in Table 5. Note in Figure 21 that the critical values of the imperfection size provided by the criteria of Fage (1943) and Carmichael (1959) are independent of the Mach number (while they depend on the chord Reynolds number), whereas the values of the transition Reynolds number - as well as their variations as a function of $h / a$-were predicted in Masad and Iyer (1993) to be dependent on the Mach number. The filled and open violet symbols correspond to the transition Reynolds numbers obtained in Masad and Iyer (1993) at $\mathrm{M}=0.8$ and 0.35 , respectively. (Since data were not available at $\mathrm{M}=0.35, \mathrm{Re}_{x \mathrm{~T}}$ was linearly interpolated from the values computed at $\mathrm{M}=0.3$ and 0.4.) As can be seen from these two data sets, the transition Reynolds number was predicted to increase at larger Mach numbers for bump height-to-length ratios up to $h / a=0.015$, while it remained essentially unchanged at larger values of $h / a$. A similar effect of compressibility on bump-induced transition was predicted in Masad and Nayfeh (1992) and in Masad and Malik (1995). As discussed in these earlier investigations, compressibility has two major effects on boundary-layer stability in the presence of bumps: first, the amplification of boundary-layer disturbances at larger subsonic Mach numbers is weakened in the regions at a sufficient distance from the bump; secondly, an increase in the Mach number leads to a strengthening of the bump-induced variations in the surface pressure distribution (see also Figure 15). In particular, the pressure gradient on the downstream side of the bump becomes more adverse, and this leads to an enhancement of the local amplification of the boundary-layer disturbances. Moreover, for a given value of $h / a$, the boundary layer is more likely to separate at larger Mach numbers because of the more adverse pressure gradient. If separation occurs, amplification of boundary-layer disturbances is strongly enhanced, as compared to the case of attached flow over the downstream side of the bump. Even if separation occurs at all Mach numbers examined for a certain value of $h / a$, the local disturbance amplification is expected to be more marked at larger Mach numbers, since the size of the separated flow region increases with increasing Mach number. Therefore, the overall effect of compressibility on bump-induced transition depends on which of these two counteracting effects is predominant. In Masad and Nayfeh (1992), Masad and Iyer (1993) and Masad and Malik (1995), the net effect of compressibility was found to be stabilizing; however, this favorable influence was shown in Masad and Iyer (1993) to become weaker at larger values of $h / a$. This trend can be also seen in Figure 21 (violet symbols). 
Revision 1. Submitted to the Special Issue of Experimental Thermal and Fluid Science (ETFS) dedicated to the $5^{\text {th }}$ International Conference on Experimental Fluid Mechanics (ICEFM)

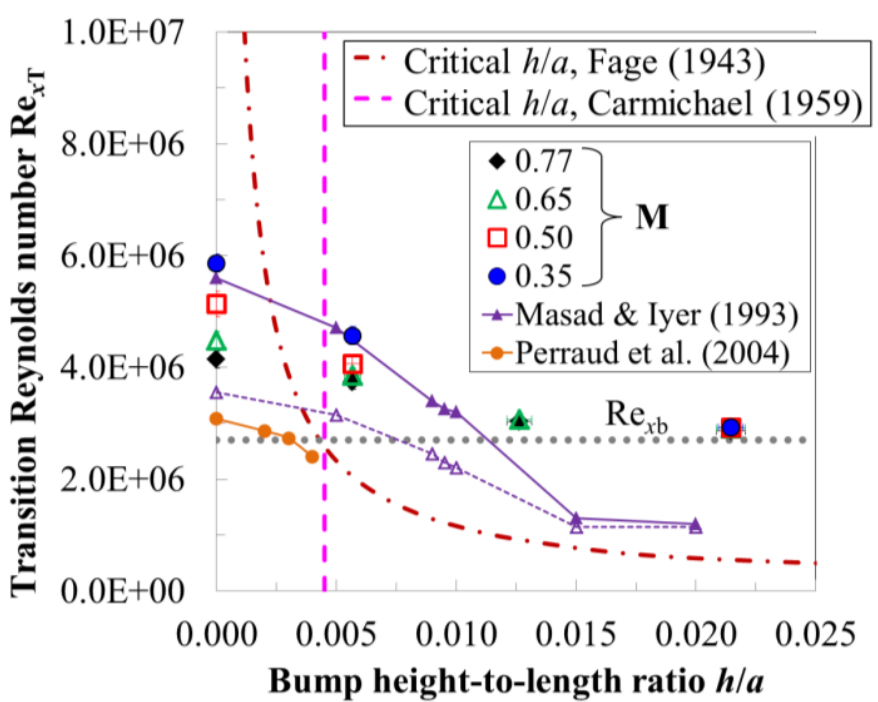

Figure 21: Transition Reynolds number as a function of the bump height-to-length ratio for $\operatorname{Re}=6 \cdot 10^{6}$ and $0.060 \leq \beta_{\mathrm{H}} \leq 0.063$.

Table 5: Bump height relative to local boundary-layer displacement thickness for the data points of Figure 21.

\begin{tabular}{|c|c|c|c|}
\hline $\mathbf{M}$ & $\begin{array}{c}\boldsymbol{h} / \boldsymbol{\delta}_{\mathbf{1 , b}} \\
(\boldsymbol{h} / \boldsymbol{a}=\mathbf{0 . 0 0 6})\end{array}$ & $\begin{array}{c}\boldsymbol{h} / \boldsymbol{\delta}_{\mathbf{1 , b}} \\
(\boldsymbol{h} / \boldsymbol{a}=\mathbf{0 . 0 1 3})\end{array}$ & $\begin{array}{c}\boldsymbol{h} / \boldsymbol{\delta}_{\mathbf{1 , b}} \\
(\boldsymbol{h} / \boldsymbol{a}=\mathbf{0 . 0 2 1})\end{array}$ \\
\hline 0.77 & 1.11 & 2.48 & 4.21 \\
\hline 0.65 & 1.13 & 2.52 & 4.27 \\
\hline 0.50 & 1.20 & 2.67 & 4.54 \\
\hline 0.35 & 1.22 & 2.71 & 4.61 \\
\hline
\end{tabular}

In the present work, larger transition Reynolds numbers were measured at smaller Mach numbers for the smooth and small-bump configurations, a trend opposite to that expected from linear stability theory. As discussed in Sec. 4.3, this effect was very likely due to the increased level of external disturbances in DNW-KRG and to the larger wall temperature ratio $T_{w} / T_{a w}$ at larger Mach numbers. In the case of the small bump, the more marked adverse pressure gradient on the downstream side of the bump found at larger Mach numbers may have also contributed to the observed trend. Spectra of total pressure fluctuations $p_{0}^{\prime}$ in DNW-KRG were measured in Koch (2004) for different Mach numbers; the variation of $p_{0}^{\prime}$ as a function of the frequency $f$ and of the Mach number $M$ was approximated in Risius et al. (2018) and Risius (2018) by the following relation:

$$
p_{0}^{\prime} / \bar{p}_{0}=f^{0.036 \cdot \mathrm{M}-0.458} \cdot 10^{1.537 \cdot \mathrm{M}-3.462}
$$

where $\bar{p}_{0}$ is the average value of the total pressure. Nevertheless, the sensitivity of the transition Reynolds number to variations in the Mach number was observed in the present work to weaken for increasing values of $h / a$ (see Figure 21). This also resulted in a more pronounced reduction of the transition Reynolds number with increasing $h / a$ for smaller Mach numbers. In contrast, the reduction in $\operatorname{Re}_{x \mathrm{~T}}$ as a function of $h / a$ observed for the data from Masad and Iyer (1993) was more marked at larger Mach numbers. These different trends may be due to a number of factors not directly related to compressibility effects: besides the aforementioned variations of disturbance environment and wall temperature ratio as function of the Mach number (which are specific to DNW-KRG), other factors influencing bump-induced transition are among others - the bump shape and the bump location. In particular, the bump contour considered in Masad and Iyer (1993) in the vicinity of the bump start/end and of the bump crest was slightly steeper and flatter, respectively, than that examined in the present work. This presumably influenced the evolution of 
Revision 1. Submitted to the Special Issue of Experimental Thermal and Fluid Science (ETFS) dedicated to the $5^{\text {th }}$ International Conference on Experimental Fluid Mechanics (ICEFM)

the bump-induced flow variation (and boundary-layer disturbance amplification) as a function of the Mach number. Moreover, the value of the Reynolds number at the bump location was $\operatorname{Re}_{x b}=1 \cdot 10^{6}$ in Masad and Iyer (1993), i.e., smaller than those considered in the present work. The value of $\operatorname{Re}_{x b}$ was shown in Masad and Iyer (1993) and Masad and Malik (1995) to have a significant impact on the behavior of bumpinduced transition.

In the present study, the compressibility effect on bump-induced transition could not be examined independently from the aforementioned parameters. In fact, the application of a method to "reveal" the effect of compressibility on transition, such as that presented in Risius et al. (2018) and Risius (2018) for a smooth surface, is precluded here by the lack of quantitative information on the interaction between the bumps and the boundary-layer disturbances at different Mach numbers. Nevertheless, the trends for the variation of $\mathrm{Re}_{x \mathrm{~T}}$ as a function of $h / a$ discussed in Sec. 5.1 are confirmed in Figure 21 for all examined Mach numbers. At a bump height-to-length ratio approximately corresponding to the critical value of $h / a$ from Carmichael (1959), a significant decrease in the transition Reynolds number was observed for the present data and for those of Masad and Iyer (1993), while the critical h/a from Fage (1943) appears to qualitatively predict the reduction of $\mathrm{Re}_{x \mathrm{~T}}$ found in this work and in Masad and Iyer (1993).

\subsection{Combined Effect of Bump Height-to-Length Ratio and Chord Reynolds Number}

The influence of the bump height-to-length ratio $h / a$ on boundary-layer transition is analyzed in this section in combination with the effect of the chord Reynolds number Re. The examined test conditions are those presented in Sec. 4.4. The results obtained at the three different Reynolds numbers are summarized in Figure 22, where the transition Reynolds number is presented as a function of the bump height-tolength ratio. The data obtained at different chord Reynolds numbers are shown by symbols with different colors, while the values of $\mathrm{Re}_{x \mathrm{~b}}$ for the different chord Reynolds numbers are indicated by dotted lines with the same colors as those of the corresponding transition data. (The Reynolds number based on the bump location increased from $\operatorname{Re}_{x \mathrm{~b}}=2.7$ to $4.5 \cdot 10^{6}$ as the Reynolds number was increased from $\operatorname{Re}=6$ to $10 \cdot 10^{6}$.) The correlations between $\mathrm{Re}_{x \mathrm{~T}}$ and critical $h / a$ reported by Fage (1943) are shown by dasheddotted lines, whereas the critical bump-to-height ratios derived from the criterion provided by Carmichael (1959) are indicated by dashed lines. In these earlier works, it had been already found that an increase in the chord Reynolds number led to a decrease in the allowable size of imperfections for maintenance of laminar flow. This effect is accounted for in Eqs. (I) and (II) and shown in Figure 22 by dashed-dotted and dashed lines with different colors, which match those of the corresponding chord Reynolds numbers. The values of the bump height $h$ relative to the local boundary-layer displacement thickness $\delta_{1, \mathrm{~b}}$ are reported in Table 6 for the corresponding data points of Figure 22.

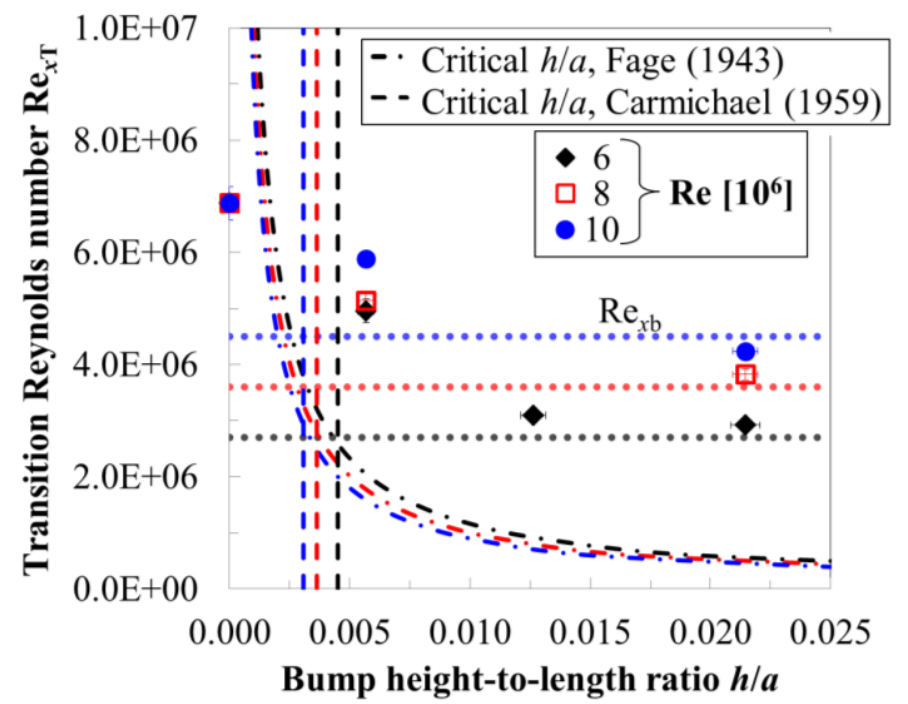

Figure 22: Transition Reynolds number as a function of the bump height-to-length ratio for $\mathrm{M}=0.77$ and $\beta_{\mathrm{H}}=0.096$. 
Revision 1. Submitted to the Special Issue of Experimental Thermal and Fluid Science (ETFS) dedicated to the $5^{\text {th }}$ International Conference on Experimental Fluid Mechanics (ICEFM)

Table 6: Bump height relative to local boundary-layer displacement thickness for the data points of Figure 22.

\begin{tabular}{|c|c|c|c|}
\hline $\operatorname{Re}\left[\mathbf{1 0}^{\mathbf{6}}\right]$ & $\begin{array}{c}\boldsymbol{h} / \boldsymbol{\delta}_{\mathbf{1 , b}} \\
(\boldsymbol{h} / \boldsymbol{a}=\mathbf{0 . 0 0 6})\end{array}$ & $\begin{array}{c}\boldsymbol{h} / \boldsymbol{\delta}_{\mathbf{1 , b}} \\
(\boldsymbol{h} / \boldsymbol{a}=\mathbf{0 . 0 1 3})\end{array}$ & $\begin{array}{c}\boldsymbol{h} / \boldsymbol{\delta}_{\mathbf{1 , b}} \\
(\boldsymbol{h} / \boldsymbol{a}=\mathbf{0 . 0 2 1})\end{array}$ \\
\hline 6 & 1.15 & 2.56 & 4.34 \\
\hline 8 & 1.33 & 2.97 & 5.03 \\
\hline 10 & 1.46 & 3.26 & 5.54 \\
\hline
\end{tabular}

As presented in Sec. 4.4, bump-induced transition was observed in the present work to occur at a more upstream location at larger chord Reynolds numbers. This finding is in line with the experimental results of Fage (1943) and Carmichael (1959), and also with the predictions of Masad and Iyer (1993) and Masad and Malik (1995). However, the transition Reynolds number was seen to increase at larger values of Re for both small and big bumps configurations (i.e., for $h / a=0.006$ and 0.021 , respectively). The reason for the increase of $\operatorname{Re}_{x \mathrm{~T}}$ with increasing chord Reynolds number at $h / a=0.021$ is that transition occurred over the downstream side of the big bump. As shown in Sec. 4.4, the upstream shift in the transition location for an increase in the chord Reynolds number from $\mathrm{Re}=6$ to $8 \cdot 10^{6}$ was small in the case of the big bump; the transition Reynolds number thus scaled almost linearly with Re. This behavior is in line with the predictions of Masad and Iyer (1993) and Masad and Malik (1995) for transition occurring over the downstream side of a big bump, where boundary-layer separation was enhanced at larger unit Reynolds numbers $U_{\infty} / v_{\infty}$. (At $\operatorname{Re}=10 \cdot 10^{6}$, for the reasons discussed in Sec. 4.4, transition was detected in the present work upstream of the bump crest; the corresponding data point in Figure 22 is therefore below the blue dotted line indicating the value of $\mathrm{Re}_{x \mathrm{~b}}$.) The behavior observed at $h / a=0.006$ appears to be in contrast to the predictions of Masad and Iyer (1993) and Masad and Malik (1995), where $\mathrm{Re}_{x \mathrm{~T}}$ was found to decrease at larger unit Reynolds numbers (for fixed bump location $x_{\mathrm{b}}$ ), provided that transition occurred downstream of the bump end. Moreover, the present experimental observations seem to be also in contrast to the expectation of a more pronounced impact on transition of bumps with larger relative bump height $h / \delta_{1, \mathrm{~b}}$ (as given here by larger Re, see Table 6). One possible reason for the difference between the evolution of $\operatorname{Re}_{x \mathrm{~T}}$ as a function of Re observed here at $h / a=0.006$ and that found in Masad and Iyer (1993) and Masad and Malik (1995) for small bumps may be the different value of the bump location. In fact, the value of $x_{\mathrm{b}}$ considered in those previous numerical studies was smaller than that examined in the present work; in particular, the range of $\mathrm{Re}_{x \mathrm{~b}}$ at which transition occurred downstream of the bump end in Masad and Iyer (1993) and Masad and Malik (1995) was below $\operatorname{Re}_{x b}=1.8 \cdot 10^{6}$, i.e., below the values of $\mathrm{Re}_{x \mathrm{~b}}$ considered here. Another possible reason for the unexpected increase of $\mathrm{Re}_{x \mathrm{~T}}$ with increasing $\mathrm{Re}$ at $h / a=0.006$ may be the "unit Reynolds number effect" on transition in DNW-KRG, which was discussed in Risius et al. (2018) and Risius (2018) for a smooth flat-plate configuration. As can be seen in Eq. (IV), the level of external disturbances (total pressure fluctuations) in DNW-KRG decreases at larger frequencies. At the same time, for transition occurring downstream of the bump end, the frequency of the most amplified Tollmien-Schlichting wave at $x_{\mathrm{T}} / c$ was shown in Masad and Iyer (1993) to increase at larger unit Reynolds numbers. Assuming that the receptivity process is approximately the same, the decreased level of external disturbances at larger frequencies in DNW-KRG may lead to a smaller initial amplitude of the Tollmien-Schlichting waves responsible for transition at larger Re, and thus to the observed increase in the transition Reynolds number.

\section{Conclusions}

The effect of surface bumps on boundary-layer transition was investigated in the low-turbulence Cryogenic Ludwieg-Tube Göttingen by means of the temperature-sensitive paint measurement technique. The experiments were conducted in a compressible (quasi-) two-dimensional flow at subsonic freestream Mach numbers from 0.35 to 0.77 and chord Reynolds numbers from 3.5 to $10 \cdot 10^{6}$. Various streamwise pressure gradients, relevant for natural laminar flow surfaces, were implemented. A two-dimensional flatplate model was modified to enable the installation of exchangeable inserts on the model upper side. 
Revision 1. Submitted to the Special Issue of Experimental Thermal and Fluid Science (ETFS) dedicated to the $5^{\text {th }}$ International Conference on Experimental Fluid Mechanics (ICEFM)

Quasi-two-dimensional bumps, with a sinusoidal shape in the streamwise direction, were manufactured on the inserts. The model was also equipped with pressure taps for the measurement of the surface pressure distributions. Three bumps with fixed length and different height were examined in this work.

The effect of a larger bump height-to-length ratio was to induce boundary-layer transition to occur at a more upstream location; this effect was particularly pronounced in the case of flow separation caused by the local adverse pressure gradient on the downstream side of the bump, with transition occurring even upstream of the end of the bump. The presence of a laminar separation bubble on the downstream side of the largest examined bump was verified via oil-film visualizations.

In the case of the bump with the smallest height-to-length ratio, stronger global flow acceleration led bump-induced transition to occur at a more downstream location; in contrast, transition induced by the bump with the largest height-to-length ratio was essentially unaffected by the global pressure gradient. The effect of the global flow acceleration on transition induced by the medium-sized bump was dependent on the Mach and Reynolds numbers. At larger Mach and Reynolds numbers, transition occurred in the region of adverse pressure gradient downstream of the bump crest, and the transition location remained essentially unchanged as the global pressure gradient was varied. In contrast, at smaller Mach and Reynolds numbers, the laminar boundary layer lasted over the region of local adverse pressure gradient, and the transition location showed a moderate dependency on the global pressure gradient. The sensitivity of boundary-layer transition to the influence of the bumps was found to be dependent on the global pressure gradient: the variation of the transition Reynolds number as a function of the bump height-tolength ratio was more pronounced in the case of stronger global flow acceleration. The present results show that the influence on transition of the global streamwise pressure gradient has to be accounted for to improve the reliability of criteria for allowable size of bumps on natural laminar flow surfaces, as compared to those provided in previous work.

The effect of a larger Mach number on bump-induced transition at small values of the bump height-tolength ratio was to decrease the transition Reynolds number. In a manner analogous to that observed in previous work on a smooth surface, this result was very likely due to the increase in the external disturbance level and in the wall temperature ratio at larger Mach numbers, which are specific to the used wind tunnel; however, the more marked adverse pressure gradient on the downstream side of the bump may have also contributed to the observed trend. Nevertheless, the reduction in the transition Reynolds number (with respect to its value obtained with a smooth surface) due to the effect of the small bump was less marked at larger Mach numbers. At larger values of the bump height-to-length ratio, the effect of the Mach number on boundary-layer transition was significantly weakened: it even vanished in the presence of the bump with the largest height-to-length ratio.

Larger transition Reynolds numbers were obtained with increasing chord Reynolds numbers. For large values of the bump height-to-length ratio, however, this result should be not misinterpreted: in fact, transition occurred in the adverse pressure gradient region on the downstream side of the bump at all considered chord Reynolds numbers. Since the transition location remained essentially unchanged, the transition Reynolds number scaled almost linearly with the chord Reynolds number. The increase in the transition Reynolds number at larger chord Reynolds numbers observed at small values of the bump height-to-length ratio was in contrast with the predictions of previous numerical studies. Possible reasons for the different evolution found in the present work are the more downstream bump location examined here and the "unit Reynolds number effect", already described in previous work on smooth surfaces.

\section{Acknowledgements}

This work was conducted within the German Federal Aeronautical Research Programme LuFo V-1 "Low Drag Aircraft in Operation" (LDAinOp), funded by the German Federal Ministry for Economic Affairs and Energy [grant number 20A1302B]. The authors are grateful to the German Federal Ministry for Economic Affairs and Energy for the financial support. Moreover, the authors would like to thank: L. Koop (DLR), H. Rosemann (DLR), S. Schaber (Airbus, compound project partner in LuFo V-1) and W. Kühn (formerly Airbus) for the assistance to the activities within the LuFo V-1 Research Programme; 
Revision 1. Submitted to the Special Issue of Experimental Thermal and Fluid Science (ETFS) dedicated to the $5^{\text {th }}$ International Conference on Experimental Fluid Mechanics (ICEFM)

R. Kahle, M. Aschoff, A. Grimme and S. Hucke (DNW) for the support during the measurement campaign at DNW-KRG; S. Brockmann, P. Hayen, G. Löschen, H. Tholen, R. Twele and S. von Deetzen (Deharde Maschinenbau Helmut Hoffmann $\mathrm{GmbH}$ ) for constructing and manufacturing the wind-tunnel model; V. Ondrus (University of Hohenheim) for the chemical development and synthesis of the used temperature-sensitive paint; U. Henne (DLR) for the support to the TSP data acquisition; E. Schülein (DLR) for providing the silicone oils for the oil-film visualizations, for the suggestions for successful OFV in short-duration facilities and for the discussions of the acquired OFV images; J. Lunte (DLR) for the determination of the viscosity of one of the used silicone oils; M. Pani (ITS Umbria) for the support in drafting the schematics; and M. Wüstefeld (DLR) for the model preparation before TSP application.

\section{References}

Al-Maaitah AA, Nayfeh AH, Ragab SA (1990). Effect of wall cooling on the stability of compressible subsonic flow over smooth humps and backward-facing steps. Physics of Fluids A 2(3): 381-389.

Arnal D (1992) Boundary Layer Transition: Prediction, Application to Drag Reduction. AGARD R-786: 51 to $5-59$.

Ashill PR, Betts CJ, Gaudet IM (1996) A wind tunnel study of transition flows on a swept panel wing at high subsonic speeds. CEAS $2^{\text {nd }}$ European Forum on Laminar Flow Technology: 10.3 to 10.17.

Braslow AL (1966) A Review of Factors Affecting Boundary-Layer Transition. NASA TN-D-3384.

Carmichael BH (1959). Surface waviness criteria for swept and unswept laminar suction wings. Northrop Aircraft, Inc., Report NOR-59-438 (BLC-123).

Cebeci T, Egan DA (1989). Prediction of Transition Due to Isolated Roughness. AIAA Journal 27(7): 870-875.

Costantini M, Hein S, Henne U, Klein C, Koch S, Schojda L, Ondrus V, Schröder W (2016) Pressure Gradient and Non-adiabatic Surface Effects on Boundary-Layer Transition. AIAA Journal 54(11): 34653480 .

Costantini M (2016) Experimental Analysis of Geometric, Pressure Gradient and Surface Temperature Effects on Boundary-Layer Transition in Compressible High Reynolds Number Flows. PhD Thesis, RWTH Aachen.

Costantini M, Risius S, Klein C (2018) Non-Adiabatic Surface Effects on Step-Induced Boundary-Layer Transition. Flow, Turbulence and Combustion 100(4): 1145-1177.

Fage A (1943) The Smallest Size of a Spanwise Surface Corrugation which Affects Boundary-Layer Transition on an Aerofoil. ARC Reports and Memoranda 2120.

Forte M, Perraud J, Séraudie, A, Beguet, S, Gentili, L, Casalis G (2015). Experimental and Numerical Study of the Effect of Gaps on Laminar Turbulent Transition of Incompressible Boundary Layers. Procedia IUTAM 14C: 448-458.

Franco JA, Hein S, Valero E (2018) Effect of humps and indentations on boundary-layer transition of compressible flows using the AHLNS methodology. Proc. $6^{\text {th }}$ ECCM $-7^{\text {th }}$ ECFD, Paper 1821.

Hansen H (2010) Laminar Flow Technology - The Airbus View. Proc. 27 ${ }^{\text {th }}$ ICAS: 453-461.

Holmes BJ, Obara CJ (1983) Observations and Implications of Natural Laminar Flow on Practical Airplane Surfaces. Journal of Aircraft 20(12): 993-1006.

Holmes BJ, Obara CJ, Martin GL, Domack CS (1985) Manufacturing tolerances for natural laminar flow airframe surfaces. SAE Paper 850863. 
Revision 1. Submitted to the Special Issue of Experimental Thermal and Fluid Science (ETFS) dedicated to the $5^{\text {th }}$ International Conference on Experimental Fluid Mechanics (ICEFM)

Hood MJ (1939) The Effects of Surface Waviness and of Rib Stitching on Wing Drag. NACA TN 724.

Horton HP (1968) Laminar separation bubbles in two and three dimensional incompressible flow. PhD Thesis, Queen Mary Univ. London.

Kirk TM, Yarusevych S (2017) Vortex shedding within laminar separation bubbles forming over an airfoil. Experiments in Fluids 58, Article 43.

Klein C, Engler RH, Henne U, Sachs WE (2005) Application of pressure-sensitive paint for determination of the pressure field and calculation of the forces and moments of models in a wind tunnel. Experiments in Fluids 39: 475-483.

Klein C, Henne U, Sachs WE, Beifuss U, Ondrus V, Bruse M, Lesjak R, Löhr M, Becher A, Zhai J (2015) Combination of Temperature-Sensitive Paint (TSP) and Carbon Nanotubes (CNT) for Transition Detection. AIAA Paper 2015-1558.

Koch S (2004) Zeitliche und räumliche Turbulenzentwicklung in einem Rohrwindkanal und deren Einfluss auf die Transition an Profilmodellen. DLR FB 2004-19.

Kreplin H-P, Höhler G (1992) Application of the Surface Hot Film Technique for Laminar Flow Investigations," ${ }^{\text {st }}$ European Forum on Laminar Flow Technology: 123-131.

Lee T, Nonomura T, Asai K, Liu T (2018) Linear least-squares method for global luminescent oil film skin friction field analysis. Review of Scientific Instruments 89, Article 065106.

Lemarechal J, Klein C, Henne U, Puckert DK, Rist U (2018) Detection of Lambda- and Omega-vortices with the Temperature-Sensitive Paint method in the late stage of controlled laminar-turbulent transition. Submitted to Experiments in Fluids.

Liu T, Sullivan JP (2005) Pressure and temperature sensitive paint. Springer-Verlag, Berlin.

Masad JA, Iyer V (1993) Transition Prediction and Control in Subsonic Flow Over a Hump. NASA CR 4543.

Masad JA, Malik MR (1995) Link Between Flow Separation and Transition Onset. AIAA Journal 33(5): 882-887.

Merzkirch W (1987) Techniques of Flow Visualization. AGARDograph 302.

Meyer F, Kleiser L (1989) Numerical Investigation of Transition in 3D Boundary Layers. AGARD CP438: $16-1$ to $16-17$.

Miozzi M, Capone A, Costantini M, Fratto L, Klein C, Di Felice F (2019) Skin friction and coherent structures within a laminar separation bubble. Experiments in Fluids 60, Article 13.

Nayfeh AH, Ragab SA, Al-Maaitah AA (1988) Effect of Bulges on the Stability of Boundary Layers. Physics of Fluids 31(4): 796-806.

Ondrus V, Meier R, Klein C, Henne U, Schäferling M, Beifuss U (2015) Europium 1,3di(thienyl)propane-1,3-diones with Outstanding Properties for Temperature Sensing. Sensors and Actuators A: Physical 233: 434-441.

Perraud J, Arnal D, Séraudie A, Tran D (2004) Laminar-turbulent Transition on Aerodynamic Surfaces with Imperfections. RTO-AVT-111: 14-1 to 14-14.

Risius S (2018) Development of a time-resolved quantitative surface-temperature measurement technique and its application in short-duration wind tunnel testing. PhD Thesis, Georg-August University Göttingen.

Risius S, Costantini M, Koch S, Hein S, Klein C (2018) Unit Reynolds number, Mach number and pressure gradient effects on laminar-turbulent transition in two-dimensional boundary layers. Experiments in Fluids 59, Article 86. 
Revision 1. Submitted to the Special Issue of Experimental Thermal and Fluid Science (ETFS) dedicated to the $5^{\text {th }}$ International Conference on Experimental Fluid Mechanics (ICEFM)

Rosemann H (1997) The Cryogenic Ludwieg-Tube Tunnel at Göttingen. AGARD R-812: 8-1 to 8-13.

Schlichting H, Gersten K (2000) Boundary-Layer Theory, $8^{\text {th }}$ edition. Springer-Verlag, Berlin Heidelberg. Chap. 15: Onset of Turbulence (Stability Theory).

Schülein E (2004) Optical Skin Friction Measurements in Short-duration Facilities. AIAA Paper 20042115 .

Selig MS, Deters RW, Williamson GA (2011) Wind Tunnel Testing Airfoils at Low Reynolds Numbers. AIAA Paper 2011-0875.

Tropea C, Yarin AL, Foss JF (2007) Springer Handbook of Experimental Fluid Mechanics. SpringerVerlag, Berlin Heidelberg. Chap. 7.4: Transition-Detection by Temperature-Sensitive Paint.

Wagner RD, Bartlett DW, Collier Jr. FS (1989) Laminar Flow - The Past, Present, and Prospects. AIAA Paper 1989-0989.

Wörner A, Rist U, Wagner S (2002) Influence of humps and steps on the stability characteristics of a 2D laminar boundary layer. AIAA Paper 2002-0139. 